\title{
Holomorphic versus algebraic equivalence for deformations of real-algebraic Cauchy-Riemann manifolds
}

\author{
Bernhard LAMEL AND Nordine MiR
}

\begin{abstract}
We consider (small) algebraic deformations of germs of realalgebraic Cauchy-Riemann submanifolds in complex space and study the biholomorphic equivalence problem for such deformations. We show that two algebraic deformations of minimal holomorphically nondegenerate real-algebraic CR submanifolds are holomorphically equivalent if and only if they are algebraically equivalent.
\end{abstract}

\section{Introduction}

Since Poincaré's celebrated paper [19] published in 1907, there has been a growing literature concerned with the equivalence problem for real submanifolds in complex space (see e.g., $[4,6,7,11,13,14,22]$ for some recent works as well as the references therein). One interesting phenomenon, observed by Webster for biholomorphisms of Levi nondegenerate hypersurfaces [23], is that the biholomorphic equivalence of some types of real-algebraic submanifolds of a complex space implies their algebraic equivalence.

In this paper, we show that this very phenomenon holds for algebraic deformations of germs of minimal holomorphically nondegenerate realalgebraic CR submanifolds in complex space. Let us recall that a germ of a real-algebraic $\mathrm{CR}$ submanifold $(M, p) \subset\left(\mathbb{C}^{n}, p\right)$ is minimal if there exists no proper CR submanifold $N \subset M$ through $p$ of the same $\mathrm{CR}$ dimension as $M$. It is holomorphically nondegenerate if there exists no nontrivial holomorphic vector field tangent to $M$ near $p$ (see [21]).

An algebraic deformation of $(M, p)$ is a real-algebraic family of germs at $p$ of real-algebraic CR submanifolds $\left(M_{s}, p\right)_{s \in \mathbb{R}^{k}}$ in $\mathbb{C}^{n}$, defined for $s \in \mathbb{R}^{k}$ near 0 , such that $M_{0}=M$. We say that two such deformations $\left(M_{s}, p\right)_{s \in \mathbb{R}^{k}}$ and $\left(N_{t}, p^{\prime}\right)_{t \in \mathbb{R}^{k}}$ are biholomorphically equivalent if there exists a germ of a realanalytic diffeomorphism $\varphi:\left(\mathbb{R}^{k}, 0\right) \rightarrow\left(\mathbb{R}^{k}, 0\right)$ and a holomorphic submersion $B:\left(\mathbb{C}_{z}^{n} \times \mathbb{C}_{u}^{k},(p, 0)\right) \rightarrow\left(\mathbb{C}^{n}, p^{\prime}\right)$ such that $z \mapsto B(z, s)$ is a biholomorphism sending $\left(M_{s}, p\right)$ to $\left(N_{\varphi(s)}, p^{\prime}\right)$ for all $s \in \mathbb{R}^{k}$ close to 0 . We shall say that such 
a pair $(B, \varphi)$ is a biholomorphism between the two deformations ${ }^{1}$. We also say that they are algebraically equivalent if one can choose $\varphi$ and $B$ to be furthermore algebraic. Our main result is as follows.

Theorem 1.1. Two algebraic deformations of minimal holomorphically nondegenerate real-algebraic $C R$ submanifolds of $\mathbb{C}^{n}$ are algebraically equivalent if and only if they are biholomorphically equivalent.

For a completely trivial deformation (i.e., $k=0$ ), Theorem 1.1 is actually a consequence of the algebraicity theorem of Baouendi et al. [2], where they prove that every local biholomorphism sending holomorphically nondegenerate and minimal real-algebraic generic submanifolds of $\mathbb{C}^{n}$ must necessarily be algebraic. This is not necessarily true for biholomorphisms between deformations, even for constant ones, as the following example shows.

Example 1.1. Consider the Lewy hypersurface $M$ in $\mathbb{C}_{(z, w)}^{2}$ defined by $\operatorname{Im} w=|z|^{2}$, and consider the trivial deformation $M_{s}=M$ for $s \in \mathbb{R}^{k}$. A biholomorphic map of $\left(M_{s}, 0\right)_{s \in \mathbb{R}^{k}}$ to itself which is not algebraic is e.g., given by $\varphi(s)=s, B(z, w, s)=\left(e^{s} z, e^{2 s} w\right)$.

The main point of this example is that one cannot expect a biholomorphism between two deformations to be algebraic. However, in Example 1.1, all the "fibers" $M_{s}$ of the deformations are the same. It is not difficult to show, by using the mentioned result of [2], that the conclusion of Theorem 1.1 holds when all the fibers of the deformation are algebraically equivalent. One approach which has been successful for more general deformations is to approximate a given biholomorphism by algebraic ones; Theorem 1.1 is a consequence of such an approximation statement.

Theorem 1.2. Let $\left(M_{s}, p\right)_{s \in \mathbb{R}^{k}}$ and $\left(N_{t}, p^{\prime}\right)_{t \in \mathbb{R}^{k}}$ be algebraic deformations of real-algebraic holomorphically nondegenerate minimal CR submanifolds of $\mathbb{C}^{n}$, and assume that $(B, \varphi)$ is a biholomorphism between $\left(M_{s}, p\right)_{s \in \mathbb{R}^{k}}$ and $\left(N_{t}, p^{\prime}\right)_{t \in \mathbb{R}^{k}}$. Then for every integer $\ell>0$ there exists an algebraic biholomorphism $\left(B^{\ell}, \varphi^{\ell}\right)$ between $\left(M_{s}, p\right)_{s \in \mathbb{R}^{k}}$ and $\left(N_{t}, p^{\prime}\right)_{t \in \mathbb{R}^{k}}$ which agrees with $(B, \varphi)$ up to order $\ell$ at $(p, 0)$.

Under the stronger hypothesis that $\left(M_{0}, p\right)$ is "finitely nondegenerate", Theorem 1.2 was proved by Baouendi et al. (see [7]). However, the weakening of the nondegeneracy assumption makes it impossible to use their methods.

\footnotetext{
${ }^{1}$ By slight abuse of language, we shall always identify the map $\varphi:\left(\mathbb{R}^{k}, 0\right) \rightarrow$ $\left(\mathbb{R}^{k}, 0\right)$ with its complexification from $\left(\mathbb{C}^{k}, 0\right)$ to $\left(\mathbb{C}^{k}, 0\right)$.
} 
Let us restate our results in a more geometric fashion. For this, we use the following notation. We say that two germs of real-algebraic CR submanifolds $(M, p)$ and $\left(M^{\prime}, p^{\prime}\right)$ of $\mathbb{C}^{N}$ are biholomorphically equivalent, and write $(M, p) \sim_{h}\left(M^{\prime}, p^{\prime}\right)$ if there exists a germ of a biholomorphism $H:\left(\mathbb{C}^{N}, p\right) \rightarrow$ $\left(\mathbb{C}^{N}, p^{\prime}\right)$ and a neighborhood $U$ of $p$ in $\mathbb{C}^{N}$ such that $H(M \cap U) \subset M^{\prime}$ (we shall abbreviate this by writing $H(M) \subset M^{\prime}$ ). We say that $(M, p)$ and $\left(M^{\prime}, p^{\prime}\right)$ are algebraically equivalent, and write $(M, p) \sim_{a}\left(M^{\prime}, p^{\prime}\right)$, if there exists such a biholomorphism which is furthermore algebraic.

Let us recall that if $M \subset \mathbb{C}^{N}$ is a real-algebraic CR submanifold, for every $q \in M$ there exists a unique germ of a real-algebraic submanifold $\mathcal{W}_{q}$ through $q$ with the property that every (small) piecewise differentiable curve starting at $q$, whose tangent vectors are in the complex tangent space, has its image contained in $\mathcal{W}_{q}$ (see [2]). $\mathcal{W}_{q}$ is referred to as the local CR orbit of $q$. We shall say that $(M, p)$ has constant orbit dimension if $\operatorname{dim} \mathcal{W}_{q}$ is constant for $q$ close by $p$. The geometric counterpart of Theorem 1.1 can now be stated as follows.

Theorem 1.3. Let $(M, p)$ be a germ of a holomorphically nondegenerate real-algebraic CR submanifold, which is in addition of constant orbit dimension. Assume $\left(M^{\prime}, p^{\prime}\right)$ is a germ of a real-algebraic submanifold of $\mathbb{C}^{N}$ for which $(M, p) \sim_{h}\left(M^{\prime}, p^{\prime}\right)$. Then $(M, p) \sim_{a}\left(M^{\prime}, p^{\prime}\right)$.

Also Theorem 1.3 is a consequence of an approximation theorem, which can be stated as follows.

Theorem 1.4. Let $(M, p) \subset \mathbb{C}^{N}$ be a germ of a real-algebraic $C R$ submanifold which is holomorphically nondegenerate and of constant orbit dimension. Then for every real-algebraic $C R$ submanifold $M^{\prime} \subset \mathbb{C}^{N}$ and every positive integer $\ell$, if $h:\left(\mathbb{C}^{N}, p\right) \rightarrow \mathbb{C}^{N}$ is the germ of a biholomorphic map satisfying $h(M) \subset M^{\prime}$, there exists an algebraic biholomorphism $h^{\ell}:\left(\mathbb{C}^{N}, p\right) \rightarrow$ $\mathbb{C}^{N}$ satisfying $h^{\ell}(M) \subset M^{\prime}$ which agrees with $h$ up to order $\ell$ at $p$.

Let us briefly recall why a CR submanifold of constant orbit dimension is a deformation of its CR orbits: for a germ of a real-algebraic CR manifold $(M, p)$ which is of constant orbit dimension, there exists an integer $k \in\{0, \ldots, N\}$ and a real-algebraic submersion $S:(M, p) \rightarrow\left(\mathbb{R}^{k}, 0\right)$ such that $S^{-1}(S(q))=\mathcal{W}_{q}=: M_{S(q)}$ for all $q \in M$ near $p$. The level sets of $S$ therefore foliate $M$ by minimal real-algebraic CR submanifolds, and thus $M$ is an algebraic deformation of $M_{0}$ (see [7] or Lemma 2.1). In addition, a local biholomorphism sending two such real-algebraic CR submanifolds is also a biholomorphism of the associated deformations, since CR orbits of the 
source manifold are mapped to CR orbits of the target manifold (see e.g., [3]). On the other hand, given an algebraic deformation $\left(M_{s}\right)_{s \in \mathbb{R}^{k}}$ of a minimal, real-algebraic $\mathrm{CR}$ submanifold $\left(M_{0}, 0\right) \subset\left(\mathbb{C}^{n}, 0\right)$, the manifold $(M, 0) \subset$ $\left(\mathbb{C}^{n+k}, 0\right)$ defined by $M=\left\{(z, w) \in\left(\mathbb{C}^{n+k}, 0\right): z \in M_{\operatorname{Re} w}, \operatorname{Im} w=0\right\}$ is a real-algebraic CR submanifold of constant orbit dimension. Hence the statements given by Theorem 1.2 and Theorem 1.4 are equivalent.

If one removes the assumption about holomorphic nondegeneracy in Theorem 1.4, we can still show that the conclusion of the theorem holds if we assume a certain "mild" form of holomorphic degeneracy. We shall say that a point $p$ in a real-analytic $\mathrm{CR}$ submanifold $M \subset \mathbb{C}^{N}$ is a regular point of the holomorphic foliation on $M$ if there exists an integer $k \in\{0, \ldots, N-1\}$ and a holomorphically nondegenerate real-analytic CR submanifold $\widehat{M} \subset \mathbb{C}^{N-k}$ such that $(M, p) \sim_{h}\left(\widehat{M} \times \mathbb{C}^{k}, 0\right)$. (This notion is motivated by the structure of the holomorphic foliation arising in holomorphically degenerate CR submanifolds, that is discussed in detail in Section 5). We have the following result:

Theorem 1.5. Let $M \subset \mathbb{C}^{N}$ be a connected real-algebraic $C R$ submanifold. Then the following holds:

(i) The set of all points $p \in M$ such that $p$ is a regular point of the holomorphic foliation on $M$ and $(M, p)$ is of constant orbit dimension is the complement of a closed proper real-algebraic subvariety $\Sigma_{M}$ of $M$.

(ii) For every point $p \in M \backslash \Sigma_{M}$, for every real-algebraic CR submanifold $M^{\prime} \subset \mathbb{C}^{N}$ and for every positive integer $\ell$, if $h:(M, p) \rightarrow M^{\prime}$ is the germ of a biholomorphic map satisfying $h(M) \subset M^{\prime}$, there exists an algebraic biholomorphism $h^{\ell}:(M, p) \rightarrow M^{\prime}$ satisfying $h^{\ell}(M) \subset M^{\prime}$ which agrees with $h$ up to order $\ell$ at $p$.

The proof of Theorem 1.4 is based on a careful study of some algebraic properties of local biholomorphic mappings sending (nowhere minimal) realalgebraic CR submanifolds into each other. Given $(M, p)$ as in Theorem 1.4, we may assume without loss of generality that $p=0$ and that $M$ is generic in $\mathbb{C}^{N}$. As already explained, $M$ can be viewed as a deformation of its $\mathrm{CR}$ orbits and therefore we identify $M$ with a deformation $\left(M_{s}\right)_{s \in \mathbb{R}^{k}}$ of a certain minimal holomorphically nondegenerate real-algebraic generic submanifold $M_{0} \subset \mathbb{C}_{z}^{N-k}$ passing through 0 . The first step of the proof is to determine the dependence of a given biholomorphic map $h=h(z, u)$ with respect to the "parameter" $u \in \mathbb{C}^{k}$. Indeed, by the algebraicity theorem proved in [2], one already knows that for every fixed $s \in \mathbb{R}^{k}$ small enough, the map $z \mapsto h(z, s)$ 
is algebraic. We prove that there exists an integer $m_{0}$ such the holomorphic map $(z, u) \mapsto h(z, u)$ depends algebraically on the functions $z, u$ and $\mathcal{H}(u):=$ $\left(\left(\partial^{\gamma} h\right)(0, u) ;|\gamma| \leq m_{0}\right)$. This kind of parameter dependence result can not be obtained by using the techniques of $[2,7]$. It is obtained as a combination of some previous results of the second author $[17,18]$ and a key algebraic property, Proposition 3.1, proved in Section 3.

Once Proposition 4.1 is combined with Proposition 3.1, one obtains a system of (holomorphic) polynomial equations over $\mathbb{C}_{z}^{N-k} \times \mathbb{C}_{u}^{k}$ satisfied by the mappings $h$ and $\mathcal{H}$. At this point one could apply Artin's approximation theorem [1] to approximate $(h, \mathcal{H})$ by algebraic mappings. However, the sequence of algebraic mappings approximating $h$ need not send $M$ to $M^{\prime}$. This problem is overcome by constructing another polynomial system of real-algebraic equations over $\mathbb{R}^{k}$ fulfilled by the mapping $\mathcal{H}$. An application of a more refined version of Artin's approximation theorem due to Popescu [20] to the new polynomial system coupled with the first one (more precisely to its restriction to $\mathbb{R}^{N}$ ) provides the desired conclusion.

To derive Theorem 1.5, one needs to study holomorphically degenerate real-analytic CR manifolds and understand the structure of the holomorphic foliation (with singularities) arising from the existence of holomorphic vector fields tangent to them. This is done in Section 5 where we, in addition, show that if the manifolds are algebraic, the holomorphic foliation is algebraic.

The paper is organized as follows. Section 3 is devoted to the proof of an algebraic property for certain holomorphic mappings whose restriction on a nowhere minimal real-algebraic CR manifold satisfy some special type of polynomial identity. In Section 4, we prove the main approximation result of the paper, Theorem 4.1; Theorems 1.1 and 1.2 to 1.4 are direct consequences of this result. In Section 5, we recall several basic facts about the structure of the holomorphic foliation (with singularities) on a (holomorphically degenerate) real-analytic $\mathrm{CR}$ submanifold $M \subset \mathbb{C}^{N}$. If $M$ is real-algebraic and connected, we show that this foliation is algebraic; in particular, the singular locus of this foliation, which coincides with the complement of the set of regular points of the foliation, is a closed proper real-algebraic subvariety of $M$. We then deduce in Section 6 Theorem 1.5 from Theorem 4.1 and the results of Section 5. The basic background on CR analysis needed throughout the paper may be found e.g., in the books [3, 9].

\section{Preliminaries and notation}

We start by recalling some basic facts and introducing our notation. 


\subsection{Algebraic functions and mappings}

Throughout the paper, $\mathbb{C}\{x\}$ denotes the ring of convergent power series with complex coefficients in the variables $x=\left(x_{1}, \ldots, x_{r}\right), r \geq 1$. The ring $\mathbb{C}\{x\}$ can be identified with the ring of germs of holomorphic functions at 0 in $\mathbb{C}^{r}$, and we shall do so freely. Given any convergent power series $\eta=$ $\eta(x) \in \mathbb{C}\{x\}$, we denote by $\bar{\eta}=\bar{\eta}(x)$ the convergent power series obtained from $\eta$ by taking complex conjugates of its coefficients.

An element $f \in \mathbb{C}\{x\}$ is algebraic (or Nash) if it satisfies a nontrivial polynomial identity with polynomial coefficients, i.e., if it is algebraic over the subring $\mathbb{C}[x] \subset \mathbb{C}\{x\}$. We denote the subring of $\mathbb{C}\{x\}$ of all algebraic power series by $\mathcal{N}\{x\}$. To be completely explicit, this means that $f \in \mathcal{N}\{x\}$ if $f \in \mathbb{C}\{x\}$ and there exist polynomials $p_{j} \in \mathbb{C}[x]$ for $j=0, \ldots, m$ with $p_{m} \neq$ 0 such that

$$
\sum_{j=0}^{m} p_{j}(x) f(x)^{j}=0 .
$$

Given nonnegative integers $k$ and $s$, a germ of a holomorphic map $\left(\mathbb{C}^{k}, 0\right) \rightarrow$ $\mathbb{C}^{s}$ is algebraic if all of its components are algebraic. We also have to consider the ring of germs at 0 in $\mathbb{R}^{r}$ of (complex-valued) real-algebraic functions. This ring will be denoted by $\mathcal{N}^{\mathbb{R}}\{x\}$ and coincides with the ring of germs at 0 in $\mathbb{R}^{r}$ of (complex-valued) real-analytic functions whose complexification belongs to $\mathcal{N}\{x\}$.

\subsection{CR orbits, normal coordinates and iterated Segre mappings}

Let $M \subset \mathbb{C}^{N}$ be a real-analytic $\mathrm{CR}$ submanifold and $T^{0,1} M$ its $\mathrm{CR}$ bundle. We denote by $\mathcal{G}_{M}$ the Lie algebra generated by the sections of $T^{0,1} M$ and its conjugate $T^{1,0} M$, and by $\mathcal{G}_{M}(p) \subset \mathbb{C} T_{p} M$ the space of the evaluations at $p$ of these sections. By a theorem of Nagano (see e.g., $[3,8]$ ), for every point $p \in M$, there is a well-defined unique germ at $p$ of a real-analytic submanifold $\mathcal{W}_{p}$ satisfying $\mathbb{C} T_{q} \mathcal{W}_{p}=\mathcal{G}_{M}(q)$ for all $q \in V_{p}$. This unique submanifold is necessarily $\mathrm{CR}$ and called the local $C R$ orbit of $M$ at $p$. Note that this definition coincides with the one given in the introduction using piecewise differentiable curves running in complex tangential directions (see e.g., [3]). Since $M$ is real-analytic (resp. real-algebraic), it is easy to see that if $M$ is connected, the dimension of the local CR orbits is constant except possibly on a closed proper real-analytic (resp. real-algebraic) subvariety $\Sigma_{M}^{1}$ of $M$ (see e.g., $[6,7]$ ). If $\operatorname{dim} \mathcal{W}_{p}=\operatorname{dim} M$ for some point $p \in M$, we say that $M$ is minimal (or also of finite type) at $p$. 
Let $M$ be a generic real-algebraic submanifold of $\mathbb{C}^{N}$ of $\mathrm{CR}$ dimension $n$ and codimension $d$. We recall that a point $p \in M$ is of constant orbit dimension if it has an open neighborhood $U$ in $M$ such that $\operatorname{dim} \mathcal{W}_{q}$ is constant for $q \in U$. In this case, one may describe the obtained algebraic foliation by $\mathrm{CR}$ orbits in terms of normal coordinates as follows. First recall that coordinates $Z=(z, \eta) \in \mathbb{C}^{n} \times \mathbb{C}^{d}$ are normal coordinates for $(M, p)$ if $p=0$ and there exists a map $\Theta(z, \chi, \eta)$ defined in a neighborhood of $0 \in$ $\mathbb{C}^{2 n+d}$ and satisfying the normality conditions

$$
\Theta(z, 0, \eta)=\Theta(0, \chi, \eta)=\eta
$$

such that $M$ is given by $\eta=\Theta(z, \bar{z}, \bar{\eta})$. The map $\Theta$ also satisfies the reality condition

$$
\Theta(z, \chi, \bar{\Theta}(\chi, z, \eta))=\eta
$$

Lemma 2.1. ([6, Proposition 3.4] and [7, Lemma 3.4.1]). Let $(M, p) \subset$ $\mathbb{C}^{N}$ be a germ of a generic real-algebraic submanifold which is of constant orbit dimension at $p$, and denote by $c \in\{0, \ldots, d\}$ the codimension of the $C R$ orbits close by $p$ in $M$. Then there exist normal algebraic coordinates $Z=(z, \eta) \in \mathbb{C}^{n} \times \mathbb{C}^{d}, Z=(z, w, u) \in \mathbb{C}^{n} \times \mathbb{C}^{d-c} \times \mathbb{C}^{c}$, such that $M$ is given near the origin by an equation of the form

$$
\eta=(w, u)=\Theta(z, \bar{z}, \bar{\eta}):=(Q(z, \bar{z}, \bar{w}, \bar{u}), \bar{u})
$$

where $Q(z, \chi, \tau, u)$ is a $\mathbb{C}^{d-c}$-valued algebraic map near $0 \in \mathbb{C}^{n+N}$. Furthermore, there exist neighborhoods $U, V$ of the origin in $\mathbb{R}^{c}$ and $\mathbb{C}^{N-c}$ respectively such that for every $u \in U$, the real-algebraic submanifold given by

$$
M_{u}:=\{(z, w) \in V: w=Q(z, \bar{z}, \bar{w}, u)\}
$$

is generic and minimal at 0 .

A real-analytic submanifold $M$, given by $\eta=\Theta(z, \bar{z}, \bar{\eta})$, can be "complexified"; its complexification, which we denote by $\mathcal{M}$, is the germ at 0 of the complex submanifold of $\mathbb{C}^{2 N}$ given by

$$
\left\{(Z, \zeta) \in\left(\mathbb{C}^{N} \times \mathbb{C}^{N}, 0\right): \sigma=\bar{\Theta}(\chi, z, \eta)\right\}
$$

where $Z=(z, \eta) \in \mathbb{C}^{n} \times \mathbb{C}^{d}$ and $\zeta=(\chi, \sigma) \in \mathbb{C}^{n} \times \mathbb{C}^{d}$. 
In the whole paper, we always choose coordinates for our germ $(M, p)$ according to Lemma 2.1. We also need to recall the iterated Segre mappings $v_{j}$ attached to $(M, p)$ (see e.g., [6]), which we define as follows. First, to simplify notation, for any positive integer $j$, we denote by $t^{j}$ a variable lying in $\mathbb{C}^{n}$ and also introduce the variable $t^{[j]}:=\left(t^{1}, \ldots, t^{j}\right) \in \mathbb{C}^{n j}$. For $j=1$, we set $v_{1}\left(t^{1}, u\right):=\left(t^{1}, \Theta\left(t^{1}, 0,(0, u)\right)\right)$ for $t^{1} \in \mathbb{C}^{n}$ and $u \in \mathbb{C}^{c}$ sufficiently close to 0 ; for $j>1$ we inductively define $v_{j}:\left(\mathbb{C}^{n j} \times \mathbb{C}^{c}, 0\right) \rightarrow \mathbb{C}^{N}$ as follows:

$$
v_{j}\left(t^{[j]}, u\right):=\left(t^{j}, \Theta\left(t^{j}, \bar{v}_{j-1}\left(t^{[j-1]}, u\right)\right)\right) .
$$

Define $v_{0}(u):=(0, u) \in \mathbb{C}^{N}$, so that (2.6) also holds for $j=1$. From the construction, each iterated Segre mapping $v_{j}$ defines an algebraic map in a neighborhood of 0 in $\mathbb{C}^{n j} \times \mathbb{C}^{c}$. From (2.2) one obtains the identities

$$
v_{j}(0, u)=(0, u),\left.\quad v_{j+2}\left(t^{[j+2]}, u\right)\right|_{t^{j+2}=t^{j}}=v_{j}\left(t^{[j]}, u\right), \quad j \geq 0 .
$$

Furthermore, for every $j \geq 1$, the germ at 0 of the holomorphic map $\left(v_{j}, \bar{v}_{j-1}\right)$ takes its values in $\mathcal{M}$, where $\mathcal{M}$ is the complexification of $M$ as defined by $(2.5)$.

\section{A key algebraic property for holomorphic mappings}

\subsection{Statement of the algebraicity property}

In this section, we assume that $f:\left(\mathbb{C}^{N}, 0\right) \rightarrow\left(\mathbb{C}^{N^{\prime}}, 0\right)$, where $N \geq 2, N^{\prime} \geq 1$, is a germ of a holomorphic mapping and that $(M, 0) \subset \mathbb{C}^{N}$ is a germ of a real-algebraic generic submanifold of CR dimension $n$ and codimension $d$. We also assume that 0 is a point of constant orbit dimension in $M$, and that coordinates for $(M, 0)$ have been chosen according to Lemma $2.1 ; \mathcal{M}$ denotes the complexification of $M$ defined in Section 2.2. For every integer $\ell$, we define the subring $\mathcal{R}_{\ell}^{f}$ of $\mathbb{C}\{Z, \zeta\}$ to consist of power series which can be written in the form

$$
C\left(Z, \zeta,\left(\partial^{\gamma} \bar{f}(\zeta)\right)_{|\gamma| \leq \ell}\right)
$$

for some $C \in \mathcal{N}\{Z, \zeta\}\left[\left(\Lambda_{\gamma}\right)_{|\gamma| \leq \ell}\right]$, where for each $\gamma \in \mathbb{N}^{N}, \Lambda_{\gamma} \in \mathbb{C}^{N^{\prime}}$. Let $\mathcal{I}_{\mathcal{M}}$ be the ideal of $\mathbb{C}\{Z, \zeta\}$ of those convergent power series that vanish on $\mathcal{M}$. Denote by $\mathbb{C}\{\mathcal{M}\}$ the coordinate ring of $\mathcal{M}$, i.e., quotient ring $\mathbb{C}\{Z, \zeta\} / \mathcal{I}_{\mathcal{M}}$ and let $\pi_{\mathcal{M}}: \mathbb{C}\{Z, \zeta\} \rightarrow \mathbb{C}\{\mathcal{M}\}$ be the natural projection. We identify a convergent power series $J(Z)$ or $L(\zeta)$ with its image in $\mathbb{C}\{\mathcal{M}\}$ via $\pi_{\mathcal{M}}\left(\pi_{\mathcal{M}}\right.$ is injective on $\mathbb{C}\{Z\}$ since $M$ is assumed to be generic). Define $\mathcal{S}_{\ell}^{f}:=\pi_{\mathcal{M}}\left(\mathcal{R}_{\ell}^{f}\right)$. 
We now say that $f$ satisfies assumption $(\boldsymbol{\beta})$, if there exists a positive integer $\ell_{0}$ such that each component of the power series mapping $f$ (considered as an element of the ring $\mathbb{C}\{\mathcal{M}\}$ ) is algebraic over the quotient field of $\mathcal{S}_{\ell_{0}}^{f}$. To be more concrete, this means that

(अ) there exists an integer $\ell_{0}$, integers $k_{1}, \ldots, k_{N^{\prime}}$, and a family of algebraic power series $\delta_{r, s}=\delta_{r, s}\left(Z, \zeta,\left(\Lambda_{\gamma}\right)_{|\gamma| \leq \ell_{0}}\right) \in \mathcal{N}\{Z, \zeta\}\left[\left(\Lambda_{\gamma}\right)_{|\gamma| \leq \ell_{0}}\right], \quad r \in$ $\left\{1, \ldots, k_{s}\right\}, s=1, \ldots, N^{\prime}$, such that

$$
\sum_{r=0}^{k_{s}} \delta_{r, s}\left(Z, \zeta,\left(\partial^{\gamma} \bar{f}(\zeta)\right)_{|\gamma| \leq \ell_{0}}\right)\left(f_{s}(Z)\right)^{r}=0
$$

holds for $(Z, \zeta) \in \mathcal{M}$ near the origin, with $\delta_{k_{s}, s}\left(Z, \zeta,\left(\partial^{\gamma} \bar{f}(\zeta)\right)_{|\gamma| \leq \ell_{0}}\right) \not \equiv$ 0 on $\mathcal{M}$.

Our goal in this section is to prove that if (⿻) is fulfilled, then the mapping $f$ is necessarily algebraic over a certain field of convergent power series that are partially algebraic. To be more precise, we need to define the following rings.

Definition 3.1. Let $(z, w, u) \in \mathbb{C}^{n} \times \mathbb{C}^{d-c} \times \mathbb{C}^{c}$ be our fixed chosen normal coordinates. For every nonnegative integer $\ell$, let $\mathcal{A}_{\ell}^{f}$ be the subring of $\mathbb{C}\{z, w, u\}$ consisting of those convergent power series $T=T(z, w, u)$ which can be written in the form $A\left(z, w, u,\left(\partial^{|\alpha|} f(0, u)\right)_{|\alpha| \leq \ell)}\right.$ for some $A\left(z, w, u,\left(\Lambda_{\alpha}\right)_{|\alpha| \leq \ell}\right) \in \mathcal{N}\{z, w, u\}\left[\left(\Lambda_{\alpha}\right)_{|\alpha| \leq \ell}\right]$. We furthermore consider the $\operatorname{ring} \mathcal{A}^{f}$ defined by

$$
\mathcal{A}^{f}:=\bigcup_{j=0}^{\infty} \mathcal{A}_{j}^{f}
$$

Note that the rings $\mathcal{A}_{\ell}^{f}$ depend on the (fixed) choice of normal coordinates. We can now state the main result of this section.

Proposition 3.1. Let $(M, 0) \subset \mathbb{C}^{N}$ be a real-algebraic generic submanifold which is of constant orbit dimension at 0 , and $f:\left(\mathbb{C}^{N}, 0\right) \rightarrow\left(\mathbb{C}^{N^{\prime}}, 0\right)$ be a germ of a holomorphic mapping. If $f$ satisfies ( $\mathbf{3})$, then each component of the mapping $f$ is algebraic over the quotient field of $\mathcal{A}^{f}$.

The rest of this section is devoted to the proof of Proposition 3.1. 


\subsection{Algebraic dependence over quotient fields of certain rings}

The first step in the proof of Proposition 3.1 is given by the following lemma. Its proof is very similar to the proof of $[18$, Proposition 5.2].

Lemma 3.1. Let $M, f$ be as in Proposition 3.1. Then, for every multiindex $\mu \in \mathbb{N}^{N}$, each component of the mapping $\partial^{\mu} f$ is algebraic over the quotient field of $\mathcal{S}_{\ell_{0}+|\mu|}^{f}$.

Proof. For $\mu=0$, this is the content of assumption (\$). Differentiating (3.1) once, each first-order derivative of each component of the mapping $f$ is algebraic over the quotient field of the subring of $\mathbb{C}\{\mathcal{M}\}$ generated by $f$ and $\mathcal{S}_{\ell_{0}+1}^{f}$ (this follows from the chain rule; see [18, Proposition 5.2] for exactly similar arguments). Since (each component of) the mapping $f$ is already algebraic over the quotient field of $\mathcal{S}_{\ell_{0}}^{f} \subset \mathcal{S}_{\ell_{0}+1}^{f}$, this proves the proposition for all multiindices $\mu$ of length one. The conclusion for multiindices of arbitrary length follows in the same way by induction.

\subsection{Iterated Segre mappings and associated rings}

Our next step is to use the iterated Segre mappings as introduced in Section 2.2. For this, we need to introduce even more subrings. Let $j$ be a nonnegative integer. Recall that $t^{j}$ denotes a variable lying in $\mathbb{C}^{n}$ and $t^{[j]}$ stands for $\left(t^{1}, \ldots, t^{j}\right)$. For every such $j$ and every integer $\ell$, we let $\overline{\mathcal{B}}_{j, \ell}^{f}$ be the subring of $\mathbb{C}\left\{t^{[j+1]}, u\right\}$ consisting of the convergent power series of the form

$$
K\left(t^{[j+1]}, u,\left(\left(\partial^{|\alpha|} \bar{f}\right) \circ \bar{v}_{j}\left(t^{[j]}, u\right)\right)_{|\alpha| \leq \ell}\right),
$$

for some $K \in \mathcal{N}\left\{t^{[j+1]}, u\right\}\left[\left(\Lambda_{\alpha}\right)_{|\alpha| \leq \ell}\right]$. Similarly to before, we also set

$$
\overline{\mathcal{B}}_{j}^{f}:=\cup_{\ell=0}^{\infty} \overline{\mathcal{B}}_{j, \ell}^{f},
$$

For every integer $\ell$, define also the ring $\overline{\mathcal{D}}_{j, \ell}^{f}$ to be the subring of $\mathbb{C}\left\{t^{[j+1]}, u\right\}$ consisting of those power series of the form

$$
A\left(t^{[j+1]}, u,\left(\left(\partial^{|\alpha|} \bar{f}\right)(0, u)\right)_{|\alpha| \leq \ell}\right),
$$

for some $A \in \mathcal{N}\left\{t^{[j+1]}, u\right\}\left[\left(\Lambda_{\alpha}\right)_{|\alpha| \leq \ell}\right]$ and we also set $\overline{\mathcal{D}}_{j}^{f}:=\cup_{\ell=0}^{\infty} \overline{\mathcal{D}}_{j, \ell}^{f}$. 
Analogously, define the ring $\mathcal{D}_{j, \ell}^{f}$ as the subring of $\mathbb{C}\left\{t^{[j+1]}, u\right\}$ consisting of the power series of the form

$$
R\left(t^{[j+1]}, u,\left(\left(\partial^{|\alpha|} f\right)(0, u)\right)_{|\alpha| \leq \ell}\right),
$$

for some $R \in \mathcal{N}\left\{t^{[j+1]}, u\right\}\left[\left(\Lambda_{\alpha}\right)_{|\alpha| \leq \ell}\right]$ and set $\mathcal{D}_{j}^{f}:=\cup_{\ell=0}^{\infty} \mathcal{D}_{j, \ell}^{f}$.

Our next step is to prove the following result.

Lemma 3.2. Let $M, f$ be as in Proposition 3.1. With the above notation, for every multiindex $\mu \in \mathbb{N}^{N}$ and every integer $j$, each component of the mapping $\left(\partial^{\mu} f\right) \circ v_{j+1}$ is algebraic over the quotient field of $\overline{\mathcal{B}}_{j}^{f}$.

In order to prove Lemma 3.2, we need the following lemma which is a kind of "step-down" procedure for algebraicity over the rings $\overline{\mathcal{B}}_{j+1}^{f}$; it can be seen as an adaptation of [18, Lemma 5.4] to our situation.

Lemma 3.3. Assume that we are in the setting of Lemma 3.2, and let $j$ be a positive integer. Assume that $g:\left(\mathbb{C}^{N}, 0\right) \rightarrow \mathbb{C}$ is a holomorphic function such that $g \circ v_{j+2}$ is algebraic over the quotient field of $\overline{\mathcal{B}}_{j+1}^{f}$. Then $g \circ v_{j}$ is algebraic over the quotient field of $\overline{\mathcal{B}}_{j-1}^{f}$.

In what follows, to shorten the notation, we write $v_{j}$ instead of $v_{j}\left(t^{[j]}, u\right)$. Proof of Lemma 3.3. By assumption, there exist positive integers $e$ and $\ell$, and a family of power series $\delta_{r} \in \mathcal{N}\left\{t^{[j+2]}, u\right\}\left[\left(\Lambda_{\alpha}\right)_{|\alpha| \leq \ell}\right], r=0, \ldots, e$, such that

$$
\sum_{r=0}^{e} \delta_{r}\left(t^{[j+2]}, u,\left(\left(\partial^{|\alpha|} \bar{f}\right) \circ \bar{v}_{j+1}\right)|\alpha| \leq \ell\right)\left(g \circ v_{j+2}\right)^{r}=0,
$$

with $\delta_{e}\left(t^{[j+2]}, u,\left(\left(\partial^{|\alpha|} \bar{f}\right) \circ \bar{v}_{j+1}\right)_{|\alpha| \leq \ell}\right) \not \equiv 0$. Let $\nu \in \mathbb{N}^{n}$ be a multiindex of minimal length with respect to the property that there exists $r \in\{1, \ldots, e\}$ satisfying

$$
\left.\frac{\partial^{|\nu|}}{\partial\left(t^{j+2}\right)^{\nu}}\left[\delta_{r}\left(t^{[j+2]}, u,\left(\left(\partial^{|\alpha|} \bar{f}\right) \circ \bar{v}_{j+1}\right)|\alpha| \leq \ell\right)\right]\right|_{t^{j+2}=t^{j}} \not \equiv 0
$$

Applying $\frac{\partial^{|\nu|}}{\partial\left(t^{j+2}\right)^{\nu}}$ to (3.4), evaluating for $t^{j+2}=t^{j}$, and using the second identity in (2.7), we obtain that

$$
\left.\sum_{r=0}^{e} \frac{\partial^{|\nu|}}{\partial\left(t_{j+2}\right)^{\nu}}\left[\delta_{r}\left(t^{[j+2]}, u,\left.\left(\left(\partial^{|\alpha|} \bar{f}\right) \circ \bar{v}_{j+1}\right)\right|_{|\alpha| \leq \ell}\right)\right]\right|_{t^{j+2}=t^{j}}\left(g \circ v_{j}\right)^{r}=0
$$


We write

$$
\left.\widetilde{\delta}_{r}\left(t^{[j+1]}, u,\left(\left(\partial^{|\alpha|} \bar{f}\right) \circ \bar{v}_{j+1}\right)_{|\alpha| \leq \ell}\right)\right)
$$

$$
:=\left.\frac{\partial^{|\nu|}}{\partial\left(t_{j+2}\right)^{\nu}}\left[\delta_{r}\left(t^{[j+2]}, u,\left(\left(\partial^{|\alpha|} \bar{f}\right) \circ \bar{v}_{j+1}\right)|\alpha| \leq \ell\right)\right]\right|_{t^{j+2}=t^{j}},
$$

and observe that by our choice of $\nu$, there exists $\tilde{r} \in\{1, \ldots, e\}$ such that

$$
\left.\widetilde{\delta}_{r}\left(t^{[j+1]}, u,\left(\left(\partial^{|\alpha|} \bar{f}\right) \circ \bar{v}_{j+1}\right)_{|\alpha| \leq \ell}\right)\right) \not \equiv 0 .
$$

Hence, if we choose $\beta \in \mathbb{N}^{n}$ such that

$$
\left.\frac{\partial^{|\beta|}}{\partial\left(t^{j+1}\right)^{\beta}}\left[\widetilde{\delta}_{r}\left(t^{[j+1]}, u,\left(\left(\partial^{|\alpha|} \bar{f}\right) \circ \bar{v}_{j+1}\right)_{|\alpha| \leq \ell}\right)\right)\right]_{t^{j+1}=t^{j-1}} \not \equiv 0
$$

and for $0 \leq r \leq e$ write

$$
\left.\widehat{\delta}_{r}:=\frac{\partial^{|\beta|}}{\partial\left(t^{j+1}\right)^{\beta}}\left[\widetilde{\delta}_{r}\left(t^{[j+1]}, u,\left(\left(\partial^{|\alpha|} \bar{f}\right) \circ \bar{v}_{j+1}\right)|\alpha| \leq \ell\right)\right)\right]\left.\right|_{t^{j+1}=t^{j-1}},
$$

we see that each $\widehat{\delta}_{r} \in \overline{\mathcal{B}}_{j-1, \ell+|\beta|}^{f}$ and that the nontrivial relation

$$
\sum_{r=0}^{e} \widehat{\delta}_{r}\left(g \circ v_{j}\right)^{r}=0
$$

provides the desired result. The proof of the lemma is complete.

Proof of Lemma 3.2. By Lemma 3.1, for every multiindex $\mu$ and every integer $s \in\left\{1, \ldots, N^{\prime}\right\}$, there exists an integer $e(\mu, s)$, a family of power series $\Delta_{r, s}^{\mu}\left(Z, \zeta,\left(\Lambda_{\gamma}\right)_{|\gamma| \leq \ell_{0}+|\mu|}\right) \in \mathcal{N}\{Z, \zeta\}\left[\left(\Lambda_{\gamma}\right)_{|\gamma| \leq \ell_{0}+|\mu|}\right], r \in\{0, \ldots, e(\mu, s)\}$, such that for all $(Z, \zeta) \in \mathcal{M}$ (near the origin)

$$
\sum_{r=0}^{e(\mu, s)} \Delta_{r, s}^{\mu}\left(Z, \zeta,\left(\partial^{\gamma} \bar{f}(\zeta)\right)_{|\gamma| \leq \ell_{0}+|\mu|}\right)\left(\partial^{\mu} f_{s}(Z)\right)^{r}=0
$$

with

$$
\Delta_{e(\mu, s), s}^{\mu}\left(Z, \zeta,\left(\partial^{\gamma} \bar{f}(\zeta)\right)_{|\gamma| \leq \ell_{0}+|\mu|}\right) \not \equiv 0 \text { on } \mathcal{M}
$$

and where $\ell_{0}$ is given by condition (\$). For every integer $j$, the algebraic map $\left(v_{j+1}, \bar{v}_{j}\right)$ takes its values in $\mathcal{M}$ and therefore, from (3.7), we have the 
following identities

$$
\sum_{r=0}^{e(\mu, s)} \Delta_{r, s}^{\mu}\left(v_{j+1}, \bar{v}_{j},\left(\left(\partial^{\gamma} \bar{f}\right) \circ \bar{v}_{j}\right)_{|\gamma| \leq \ell_{0}+|\mu|}\right)\left(\left(\partial^{\mu} f_{s}\right) \circ v_{j+1}\right)^{r}=0
$$

In order to see that (3.9) implies that $\left(\partial^{\mu} f\right) \circ v_{j+1}$ is algebraic over the quotient field of $\overline{\mathcal{B}}_{j}^{f}$ for all $j \geq 0$, we note that by Lemma 3.3 it is enough to check this for all $j \geq d+1$. Now, if $j \geq d+1$, we claim that the map

$$
\left(\mathbb{C}^{n(j+1)} \times \mathbb{C}^{c}, 0\right) \ni\left(t^{[j+1]}, u\right) \mapsto\left(v_{j+1}\left(t^{[j+1]}, u\right), \bar{v}_{j}\left(t^{[j]}, u\right)\right),
$$

which takes values in $\mathcal{M}$, has generic $\operatorname{rank} N+n=\operatorname{dim} \mathcal{M}$; from this we conclude that for these $j$, by (3.8), the relation (3.9) is nontrivial.

We now turn to the proof of this last claim, which is a consequence of the finite type criterion in [2]. Let $M_{0}$ be the real-algebraic generic submanifold given by (2.4). Since $M_{0}$ is of finite type, by the finite type criterion in $[2]$, the map $\left(\mathbb{C}^{n(d+1)}, 0\right) \ni t^{[d+1]} \mapsto v_{d+1}\left(t^{[d+1]}, 0\right)$ is of generic rank $n+d-c$, where $d, c$ are as in Lemma 2.1. Therefore, for any $j \geq d+1$, the mapping $\left(\mathbb{C}^{n j} \times \mathbb{C}^{c}, 0\right) \ni\left(t^{[j]}, u\right) \mapsto v_{j}\left(t^{[j]}, u\right)$ is of generic rank $n+d-$ $c+c=N$. Hence for all such $j$ 's, the generic rank of the mapping $\left(\mathbb{C}^{n(j+1)} \times\right.$ $\left.\mathbb{C}^{c}, 0\right) \ni\left(t^{[j+1]}, u\right) \mapsto\left(v_{j+1}\left(t^{[j+1]}, u\right), \bar{v}_{j}\left(t^{[j]}, u\right)\right)$ is equal to $N+n=\operatorname{dim} \mathcal{M}$. The proof of Lemma 3.2 is complete.

\subsection{Proof of Proposition 3.1}

An application of Lemma 3.2 for $j=0$ yields that for all multiindices $\mu \in$ $\mathbb{N}^{N}$, each component of the mapping $\partial^{\mu} f \circ v_{1}$ is algebraic over the quotient field of $\overline{\mathcal{B}}_{0}^{f}=\overline{\mathcal{D}}_{0}^{f}$. which coincides with the quotient field of $\overline{\mathcal{D}}_{0}^{f}$ since $=\overline{\mathcal{B}}_{0}^{f}$. Therefore, the conjugate mapping $\partial^{\mu} \bar{f} \circ \bar{v}_{1}$ is algebraic over the quotient field of $\mathcal{D}_{0}^{f}$; since for all $\nu \in \mathbb{N}^{N}$, each component of the mapping $\partial^{\nu} f \circ v_{2}$ is algebraic over the quotient field of $\overline{\mathcal{B}}_{1}^{f}$ by Lemma 3.2 , the transitivity of being algebraic implies that each component of the mapping $\partial^{\nu} f \circ v_{2}$ is algebraic over the quotient field of $\mathcal{D}_{1}^{f}$. Proceeding inductively, we see that for every multiindex $\beta \in \mathbb{N}^{N}$ and every even integer $j$, the components of the map $\partial^{\beta} f \circ v_{j}$ are all algebraic over the quotient field of $\mathcal{D}_{j-1}^{f}$ and for every odd integer $j$ the same algebraicity property holds over the quotient field of $\overline{\mathcal{D}}_{j-1}^{f}$. Hence for $j=2(d+1)$, there exists positive integers $b, e$, convergent power series $\Psi_{\nu} \in \mathcal{N}\left\{t^{[2(d+1)]}, u\right\}\left[\left(\Lambda_{\gamma}\right)_{|\gamma| \leq b}\right], \nu \in\{0, \ldots, e\}$, such that a nontrivial 
relation of the following form

$$
\left.\sum_{\nu=0}^{e} \Psi_{\nu}\left(t^{[2(d+1)]}, u,\left(\left(\partial^{\gamma} f\right)(0, u)\right)_{|\gamma| \leq b}\right)\right)\left(f \circ v_{2(d+1)}\left(t^{[2(d+1)]}, u\right)\right)^{\nu}=0
$$

holds for all $\left(t^{[2(d+1)]}, u\right)$ sufficiently close to the origin.

In order to see that (3.10) implies that each component of $f$ is algebraic over the quotient field of $\mathcal{A}^{f}$, we need to invert the map $v_{2(d+1)}$. By the minimality criterion given in $[2,3]$, there exists points arbitrarily close to the origin in $\mathbb{C}^{2(d+1) n}$ such that $t^{[2(d+1)]} \mapsto v_{2(d+1)}\left(t^{[2(d+1)]}, 0\right)$ is of rank $N-c$ at those points with image the origin in $\mathbb{C}^{N}$. Pick a point $T^{0} \in$ $\mathbb{C}^{2(d+1) n}$ with the above property and such that $(3.10)$ holds near $\left(T^{0}, 0\right) \in$ $\mathbb{C}^{2(d+1) n} \times \mathbb{C}^{c}$. From the rank theorem, there exists an algebraic mapping $\theta:\left(\mathbb{C}^{N}, 0\right) \rightarrow\left(\mathbb{C}^{N-c}, T^{0}\right)$ such that $v_{2(d+1)}(\theta(z, w, u), u)=(z, w, u)$. Composing (3.10) with the obtained left inverse for $v_{2(d+1)}$, we obtain an identity of the following form

$$
\left.\sum_{\nu=0}^{e} \Psi_{\nu}\left(\theta(z, w, u), u,\left(\left(\partial^{\gamma} f\right)(0, u)\right)_{|\gamma| \leq b}\right)\right)(f(z, w, u))^{\nu}=0
$$

for $(z, w, u) \in \mathbb{C}^{N}$ sufficiently close to the origin. Furthermore, it is not difficult to see that it is possible to choose the mapping $\theta$ so that the obtained relation (3.11) is still nontrivial. Hence (3.11) shows that $f$ is algebraic over the quotient field of $\mathcal{A}^{f}$. This finishes the proof of Proposition 3.1.

\section{Proof of Theorems 1.1 and 1.2 to 1.4}

The goal of this section is to prove the following approximation theorem; Theorem 1.4 (and therefore also Theorems 1.1, 1.2, 1.3) are a direct consequence of this more general theorem, from which we will also deduce Theorem 1.5 in Section 6.

Theorem 4.1. Let $(M, p) \subset \mathbb{C}^{N}$ be a germ of a holomorphically nondegenerate real-algebraic generic submanifold, which is of constant orbit dimension at $p$. Assume that $M^{\prime} \subset \mathbb{C}^{N}$ is a real-algebraic generic submanifold, and that $h:\left(\mathbb{C}^{N}, p\right) \rightarrow \mathbb{C}^{N}$ is a germ of a holomorphic map with $h(M) \subset M^{\prime}$ and Jac $h \not \equiv 0$. Then for every positive integer $\ell$, there exists a germ of an algebraic mapping $h^{\ell}:\left(\mathbb{C}^{N}, p\right) \rightarrow \mathbb{C}^{N}$ satisfying $h^{\ell}(M) \subset M^{\prime}$ and that agrees with $h$ at $p$ up to order $\ell$. 
In order to prove Theorem 4.1, we assume without loss of generality that $p=0, p^{\prime}=h(p)=0$, and that normal coordinates $Z=(z, w, u)$ have been chosen for $M$ near 0 as in Lemma 2.1. We let $h:\left(\mathbb{C}^{N}, 0\right) \rightarrow\left(\mathbb{C}^{N}, 0\right)$ be a germ of a holomorphic map of generic full rank as in the statement of Theorem 4.1. We will need the following result from [18] that follows from an inspection of the proof of [18, Proposition 4.6].

Proposition 4.1. Let $M, M^{\prime}$ and $h$ be as above. Then the mapping $h$ satisfies assumption (\$) given in Section 3.

Combining Proposition 4.1 and Proposition 3.1 we get that each component of the mapping $h$ is algebraic over the quotient field of the $\operatorname{ring} \mathcal{A}^{h}$ defined in Definition 3.1, i.e., there exists an integer $m_{0}$ and, for every integer $s \in\{1, \ldots, N\}$, an integer $k_{s}$, and a family of convergent power series $P_{r, s}\left(Z,\left(\Lambda_{\alpha}\right)_{|\alpha| \leq m_{0}}\right) \in \mathcal{N}\{Z\}\left[\left(\Lambda_{\alpha}\right)_{|\alpha| \leq m_{0}}\right], 0 \leq r \leq k_{s}$, such that

$$
\sum_{r=0}^{k_{s}} P_{r, s}\left(Z,\left(\partial^{|\alpha|} h(0, u)\right)_{|\alpha| \leq m_{0}}\right)\left(h_{s}(Z)\right)^{r}=0,
$$

with

$$
P_{k_{s}, s}\left(Z,\left(\partial^{|\alpha|} h(0, u)\right)_{|\alpha| \leq m_{0}}\right) \not \equiv 0 \text {. }
$$

For ease of notation, we write $\mathcal{H}(u):=\left(\partial^{|\alpha|} h(0, u)\right)_{|\alpha| \leq m_{0}}$. Note that (4.1) simply means that the convergent power series mapping $(h, \mathcal{H})$ satisfies a certain polynomial system with algebraic coefficients; however, solutions of this system need not necessarily give rise to holomorphic mappings sending $(M, 0)$ to $\left(M^{\prime}, 0\right)$. The goal of the subsequent paragraphs is to build up an additional system of polynomial equations with real-algebraic coefficients satisfied by the mapping $\mathcal{H}$ that will allow us to approximate the mapping $h$ by algebraic mappings in the Krull topology.

\subsection{Construction of an appropriate system of polynomial equations}

In what follows, we use the following notation to denote coordinates on jet spaces: with $m_{0}$ as given above, we write $\Lambda=\left(\Lambda_{\alpha}\right)_{|\alpha| \leq m_{0}}$ where each $\Lambda_{\alpha} \in \mathbb{C}^{N}$ for $\alpha \in \mathbb{N}^{N}$. Similarly, we write $\Gamma=\left(\Gamma_{\alpha}\right)_{\mid \alpha\rfloor \leq m_{0}}$ with $\Gamma_{\alpha} \in \mathbb{C}^{N}$. Let us also recall that coordinates in the source space $\mathbb{C}^{N}$ split as $Z=(z, w, u) \in$ $\mathbb{C}^{n} \times \mathbb{C}^{d-c} \times \mathbb{C}^{c}$ and similarly for $\zeta \in \mathbb{C}^{N}$, where we write $\zeta=(\chi, \tau, v) \in$ $\mathbb{C}^{n} \times \mathbb{C}^{d-c} \times \mathbb{C}^{c}$. 
The following lemma will allow us to construct a suitable system of polynomial equations satisfied by the power series $\mathcal{H}(u)$. Its proof is analogous to that of [16, Lemma 6.2].

Lemma 4.1. For every real-valued polynomial $r^{\prime}=r^{\prime}\left(Z^{\prime}, \bar{Z}^{\prime}\right), Z^{\prime} \in \mathbb{C}^{N}$, there exists a nontrivial polynomial

$$
\mathcal{K}=\mathcal{K}(Z, \zeta, \Lambda, \Gamma ; X)=\sum_{\nu=0}^{\delta} B_{\nu}(Z, \zeta, \Lambda, \Gamma) X^{\nu} \in \mathcal{N}\{Z, \zeta\}[\Lambda, \Gamma][X]
$$

with the following properties:

(i) $B_{\delta}(Z, \zeta, \mathcal{H}(u), \overline{\mathcal{H}}(v)) \not \equiv 0$ for $(Z, \zeta) \in \mathcal{M}$ near the origin.

(ii) for every pair of convergent power series mappings $S(u)=$ $\left(S_{\alpha}(u)\right)_{|\alpha| \leq m_{0}}$ and $F(Z)=\left(F_{1}(Z), \ldots, F_{N}(Z)\right)$ which satisfies

$$
\sum_{r=0}^{k_{s}} P_{r, s}(Z, S(u))\left(F_{s}(Z)\right)^{r}=0
$$

for every $s \in\{1, \ldots, N\}$, with

$$
P_{k_{s}, s}(Z, S(u)) \not \equiv 0,
$$

we have that

$$
\mathcal{K}\left(Z, \zeta, S(u), \bar{S}(v) ; r^{\prime}(F(Z), \bar{F}(\zeta))\right) \equiv 0
$$

for $(Z, \zeta) \in \mathbb{C}^{2 N}$ sufficiently close to the origin.

Proof. For every $s \in\{1, \ldots, N\}$, denote by $T_{s}$ and $Y_{s}$ new indeterminates and consider

$$
\mathcal{Q}_{s}\left(Z, \Lambda ; T_{s}\right):=\sum_{r=0}^{k_{s}} P_{r, s}(Z, \Lambda) T_{s}^{r}, \quad \mathcal{R}_{s}\left(\zeta, \Gamma ; Y_{s}\right):=\sum_{r=0}^{k_{s}} \bar{P}_{r, s}(\zeta, \Gamma) Y_{s}^{r}
$$

$\mathcal{Q}_{s}$ and $\mathcal{R}_{s}$ are polynomials with algebraic coefficients. Let $\Delta$ be a sufficiently small polydisc in $\mathbb{C}^{N}$ centered at the origin so that each $P_{r, s}$ is holomorphic in $\Delta \times \mathbb{C}^{\kappa}$, where $\kappa:=N$ Card $\left\{\alpha \in \mathbb{N}^{N}:|\alpha| \leq m_{0}\right\}$. For each $s=1, \ldots, N$, denote by $L_{s}\left(\right.$ resp. $\left.\bar{L}_{s}\right)$ the zero set of $P_{k_{s}, s}\left(\operatorname{resp} . \bar{P}_{k_{s}, s}\right)$ in $\Delta \times \mathbb{C}^{\kappa}$ and let $E:=\cup_{s=1}^{N}\left(L_{s} \cup \bar{L}_{s}\right)$. For every $(Z, \Lambda) \in\left(\Delta \times \mathbb{C}^{\kappa}\right) \backslash E$, every $(\zeta, \Gamma) \in(\Delta \times$ $\left.\mathbb{C}^{\kappa}\right) \backslash E$ and every $s \in\{1, \ldots, N\}$, denote by $\sigma_{1}^{(s)}(Z, \Lambda), \ldots, \sigma_{k_{s}}^{(s)}(Z, \Lambda)$ the $k_{s}$ 
roots of the polynomial $\mathcal{Q}_{s}$ (viewed as a polynomial in $T_{s}$ ). Hence, for every $(\zeta, \Gamma) \in\left(\Delta \times \mathbb{C}^{\kappa}\right) \backslash E$ and every $s \in\{1, \ldots, N\}, \bar{\sigma}_{1}^{(s)}(\zeta, \Gamma), \ldots, \bar{\sigma}_{k_{s}}^{(s)}(\zeta, \Gamma)$ are the $k_{s}$ roots of the polynomial $\mathcal{R}_{s}$.

As in [16, Lemma 6.2], consider for $(Z, \Lambda) \in\left(\Delta \times \mathbb{C}^{\kappa}\right) \backslash E$ and $(\zeta, \Gamma) \in$ $\left(\Delta \times \mathbb{C}^{\kappa}\right) \backslash E$ the following polynomial in $X$

(4.7) $W(Z, \zeta, \Lambda, \Gamma ; X):=$

$$
\prod_{\substack{1 \leq l_{j} \leq k_{j} \\ 1 \leq n_{j} \leq N_{j}}}\left(X-r^{\prime}\left(\sigma_{n_{1}}^{(1)}(Z, \Lambda), \ldots, \sigma_{n_{N}}^{(N)}(Z, \Lambda), \bar{\sigma}_{l_{1}}^{(1)}(\zeta, \Gamma), \ldots, \bar{\sigma}_{l_{N}}^{(N)}(\zeta, \Gamma)\right)\right) .
$$

By Newton's theorem on symmetric polynomials, it follows that (4.7) can be rewritten as

$$
W(Z, \zeta, \Lambda, \Gamma ; X)=X^{\delta}+\sum_{\nu<\delta} A_{\nu}(Z, \Lambda, \zeta, \Gamma) X^{\nu}
$$

where $\delta:=\prod_{j=1}^{N} k_{i}^{2}$ and where $A_{\nu}$ is of the following form

$$
A_{\nu}(Z, \zeta, \Lambda, \Gamma):=C_{\nu}\left(\left(\frac{P_{r, s}(Z, \Lambda)}{P_{k_{s}, s}(Z, \Lambda)}, \frac{\bar{P}_{r, s}(\zeta, \Gamma)}{\bar{P}_{k_{s}, s}(\zeta, \Gamma)}\right)_{\substack{1 \leq r \leq k_{s} \\ 1 \leq s \leq N}}\right)
$$

where each $C_{\nu}$ is a polynomial of its arguments depending only on $r^{\prime}$. Let

$$
I(Z, \Lambda, \Lambda, \Gamma):=\prod_{s=1}^{N} P_{k_{s}, s}(Z, \Lambda) P_{k_{s}, s}(\zeta, \Gamma) .
$$

For a suitable integer $\varpi, \mathcal{K}(Z, \zeta, \Lambda, \Gamma ; X):=I^{\varpi} \cdot W \in \mathcal{N}\{Z, \zeta\}[\Lambda, \Gamma][X]$. We claim that the obtained polynomial $\mathcal{K}$ satisfies all desired properties. The construction of the polynomial $\mathcal{K}$ implies (ii). To prove statement (i), we note that the term $B_{\delta}$ is a sufficiently high power of the function $I$ defined in (4.10). Hence if $B_{\delta}(Z, \zeta, \mathcal{H}(u), \overline{\mathcal{H}}(v)) \equiv 0$ for $(Z, \zeta) \in \mathcal{M}$ near 0 , then there exists $s \in\{1, \ldots, N\}$ such that either $P_{k_{s}, s}(Z, \mathcal{H}(u)) \equiv 0$ for $Z \in \mathbb{C}^{N}$ close to 0 , or $\bar{P}_{k_{s}, s}(\zeta, \overline{\mathcal{H}}(v)) \equiv 0$ for $\zeta \in \mathbb{C}^{N}$ close to 0 , which is not the case by (4.2). The proof of Lemma 4.1 is complete.

Next, since $M^{\prime}$ is a real-algebraic generic submanifold of codimension $d$ through the origin, we may choose $d$ real-valued polynomials $\left(r_{1}^{\prime}, \ldots, r_{d}^{\prime}\right)$ such that $M^{\prime}$ is given near the origin by the zero set of these $d$ polynomials. Applying Lemma 4.1 for each polynomial $r_{j}^{\prime}, j=0, \ldots, d$, we obtain a 
corresponding polynomial

$$
\mathcal{K}^{j}(Z, \zeta, \Lambda, \Gamma ; X):=\sum_{\nu=0}^{\delta_{j}} B_{\nu}^{j} X^{\nu}
$$

with each $B_{\nu}^{j}(Z, \zeta, \Lambda, \Gamma) \in \mathcal{N}\{Z, \zeta\}[\Lambda, \Gamma][X], 1 \leq \nu \leq \delta_{j}$.

For every $j \in\{1, \ldots, d\}$, define

$$
\nu_{0}^{j}=\min \left\{\nu \in\left\{0, \ldots, \delta_{j}\right\}: B_{\nu}^{j}(Z, \zeta, \mathcal{H}(u), \overline{\mathcal{H}}(v)) \not \equiv 0 \text { on } \mathcal{M} \text { near } 0\right\}
$$

Since each polynomial $\mathcal{K}^{j}$ satisfies conclusion (i) of Lemma 4.1, we know that such a $\nu_{0}^{j}$ exists (it can be shown that each $\nu_{0}^{j}>0$ but this is not needed in what follows).

Using Lemma 2.1, we choose a real-algebraic parameterization $\left(\mathbb{R}^{2 N-d-c} \times \mathbb{R}^{c}, 0\right) \ni(y, u) \mapsto \varphi(y, u) \in \mathbb{C}^{N}$ of $M$ near 0 which satisfies that for each fixed $u \in \mathbb{R}^{c}$ sufficiently close to the origin, the mapping $\left(\mathbb{R}^{2 N-d-c}, 0\right) \ni y \mapsto \varphi(y, u)$ parameterizes the manifold $M_{u}$ as defined in (2.4) near the origin. Hence for all $(y, u) \in \mathbb{R}^{2 N-d-c} \times \mathbb{R}^{c}$ sufficiently close to the origin, we have

$$
B_{\nu}^{j}(\varphi(y, u), \overline{\varphi(y, u)}, \mathcal{H}(u), \overline{\mathcal{H}(u)})=0, \quad j=0, \ldots, d, \quad \nu=0, \ldots, \nu_{0}^{j}-1 .
$$

For every $j \in\{0, \ldots, d\}$, for every $\nu<\nu_{0}^{j}$, and for every multiindex $\gamma \in$ $\mathbb{N}^{2 N-d-c}$, define

$$
\Theta_{\gamma, \nu}^{j}(u, \Lambda, \bar{\Lambda}):=\left.\frac{\partial^{|\gamma|}}{\partial y^{\gamma}}\left[B_{\nu}^{j}(\varphi(y, u), \overline{\varphi(y, u)}, \Lambda, \bar{\Lambda})\right]\right|_{y=0} \in \mathcal{N}^{\mathbb{R}}\{u\}[\Lambda, \bar{\Lambda}] .
$$

Since the ring $\mathcal{N}^{\mathbb{R}}\{u\}[\Lambda, \bar{\Lambda}]$ is noetherian, there exists an integer $n_{0}$ such that the ideal generated by the $\Theta_{\gamma, \nu}^{j}(u, \Lambda, \bar{\Lambda})$ for $\gamma \in \mathbb{N}^{2 N-d-c}, \nu<\nu_{0}^{j}, j \in$ $\{0, \ldots, d\}$ coincides with that generated by the $\Theta_{\gamma, \nu}^{j}(u, \Lambda, \bar{\Lambda})$ for $|\gamma| \leq n_{0}$, $\nu<\nu_{0}^{j}, j \in\{0, \ldots, d\}$. We observe that by the construction of the mappings $\Theta_{\gamma, \nu}^{j}$ the following lemma holds.

Lemma 4.2. For $\gamma \in \mathbb{N}^{2 N-d-c}, \nu<\nu_{0}^{j}, j \in\{0, \ldots, d\}$, let $\Theta_{\gamma, \nu}^{j}$ be defined as above. Then if $\left(\mathbb{R}^{c}, 0\right) \ni u \mapsto S(u) \in \mathbb{C}^{\kappa}$ is the germ of a real-analytic mapping satisfying

$$
\Theta_{\gamma, \nu}^{j}(u, S(u), \overline{S(u)})=0
$$


for $u \in \mathbb{R}^{c}$ close to the origin and $|\gamma| \leq n_{0}, \nu<\nu_{0}^{j}, j \in\{0, \ldots, d\}$, then $S$ satisfies

$$
B_{\nu}^{j}(Z, \bar{Z}, S(u), \overline{S(u)})=0, \quad \nu=0, \ldots \nu_{0}^{j}-1, \quad j=0, \ldots, d
$$

for all $Z=(z, w, u) \in M$ sufficiently close to the origin.

We can now define the crucial system of complex-valued real-analytic equations near the origin in $\mathbb{R}_{x}^{n} \times \mathbb{R}_{t}^{d-c} \times \mathbb{R}_{u}^{c}$ to carry out the approximation:

$$
\left\{\begin{aligned}
\sum_{r=0}^{k_{s}} P_{r, s}(x, t, u, \Lambda) T_{s}^{r} & =0, \quad s=1, \ldots, N \\
\Theta_{\gamma, \nu}^{j}(u, \Lambda, \bar{\Lambda}) & =0, \quad|\gamma| \leq n_{0}, \nu<\nu_{0}^{j}, j \in\{0, \ldots, d\}
\end{aligned}\right.
$$

This system is obviously polynomial in $T=\left(T_{1}, \ldots, T_{N}\right)$ and $(\Lambda, \bar{\Lambda})$ with coefficients that are real-algebraic functions in $\mathbb{R}^{N}$. Furthermore, using (4.1) to (4.13), we know that

$$
\begin{aligned}
& T=\left(T_{1}, \ldots, T_{N}\right)=\left(h_{1}(x, t, u), \ldots, h_{N}(x, t, u)\right)=h(x, t, u), \\
& \Lambda=\mathcal{H}(u)=\left(\partial^{|\alpha|} h(0, u)\right)_{|\alpha| \leq m_{0}}
\end{aligned}
$$

is a complex-valued real-analytic solution of system (4.14).

\subsection{Appropriate solutions of the system and end of the proof of Theorem 4.1}

At this point, the next natural step would be to apply an approximation theorem due to Artin [1] providing, for every integer $\ell$, complex-valued realalgebraic solutions $h^{\ell}(x, t, u)$ and $W^{\ell}(x, t, u)$ of system (4.14), that agree up to order $\ell$ at the origin with the mappings $h(x, t, u)$ and $\mathcal{H}(u)$, respectively. This strategy is not sufficient because in order to show that the complexification of the obtained mappings $h^{\ell}$ sends a neighborhood of 0 in $M$ to $M^{\prime}$, we need the mappings $W^{\ell}$ to be independent of $(x, t)$ (as is the case for the original solution). The question whether this kind of approximation is possible actually dates back to Artin's paper [1]; it became known as the "subring condition". A positive answer in the algebraic case has been provided by Popescu [20], and this theorem allows us to get solution mappings $W^{\ell}$ which depend only on $u$. We shall only state a version of Popescu's theorem needed for the purpose of this paper. 
Theorem 4.2. (Popescu [20]) Let $(\omega, \xi) \in \mathbb{R}^{k} \times \mathbb{R}^{q}, k, q \geq 1$, and a polynomial mapping $\Phi(X, Y)=\left(\Phi_{1}(X, Y), \ldots, \Phi_{r}(X, Y)\right) \quad$ with $\quad \Phi_{j}(X, Y) \in$ $\mathcal{N}^{\mathbb{R}}\{\omega, \xi\}[X, Y]$ for $j=1, \ldots, r, X=\left(X_{1}, \ldots, X_{p}\right), Y=\left(Y_{1}, \ldots, Y_{m}\right)$. Suppose that there exists formal power series mappings $x(\omega) \in$ $(\mathbb{R}[[\omega]])^{p}, y(\omega, \xi) \in(\mathbb{R}[[\omega, \xi]])^{m}$ safisfying $\Phi(x, y)=0$. Then for every integer $\ell$, there exists $x^{\ell} \in\left(\mathcal{N}^{\mathbb{R}}\{\omega\}\right)^{p}, y^{\ell} \in\left(\mathcal{N}^{\mathbb{R}}\{\omega, \xi\}\right)^{m}$ satisfying $\Phi\left(x^{\ell}, y^{\ell}\right)=0$ such that $x^{\ell}$ and $y^{\ell}$ agree at 0 up to order $\ell$ with $x$ and $y$ respectively.

Remark 4.1. The fact that the system of polynomial equations has algebraic coefficients in the above result is of fundamental importance. Indeed, the analogous statement for polynomial systems with analytic coefficients does not hold in general, as a well-known example due to Gabrielov [10] shows.

Applying Theorem 4.2 to the real equations associated to the system of polynomial equations given by (4.14), we obtain, for every positive integer $\ell$, a real-analytic $\mathbb{C}^{N}$-valued algebraic mapping $h^{\ell}=h^{\ell}(x, t, u)$ and a real-analytic $\mathbb{C}^{\kappa}$-valued algebraic mapping $W^{\ell}=W^{\ell}(u)$, both defined in a neighborhood of $0 \in \mathbb{R}^{N}$ (depending on $\ell$ ) and agreeing with $h(x, t, u)$ and $\mathcal{H}(u)$ up to order $\ell$ at 0 respectively. We complexify the mappings $h^{\ell}$ and $W^{\ell}$ without changing the notation. Since each mapping $h^{\ell}=h^{\ell}(Z)$ is algebraic and agrees with the mapping $h=h(Z)$ up to order $\ell$ at 0 , the proof of Theorem 4.1 is completed by the following lemma.

Lemma 4.3. In the above setting and with the above notation, for $\ell$ sufficiently large, the holomorphic map $h^{\ell}$ sends a neighborhood of 0 in $M$ to $M^{\prime}$.

Proof of Lemma 4.3. Recall first that $\left(r_{1}^{\prime}, \ldots, r_{d}^{\prime}\right)$ are $d$ real-valued polynomials such that $M$ is given near the origin by the zero set of these $d$ polynomials. Suppose, by contradiction, that the conclusion of the lemma does not hold. Considering a subsequence if necessary, we may assume, without loss of generality, that for every integer $\ell$,

$$
r_{1}^{\prime}\left(h^{\ell}(Z), \overline{h^{\ell}}(\zeta)\right) \not \equiv 0,(Z, \zeta) \in \mathcal{M}, \text { near } 0 .
$$

Let

$$
\mathcal{K}^{1}(Z, \zeta, \Lambda, \Gamma ; X)=\sum_{\nu=0}^{\delta_{1}} B_{\nu}^{1}(Z, \zeta, \Lambda, \Gamma) X^{\nu}
$$

be the polynomial given by Lemma 4.1 associated to the real polynomial $r_{1}^{\prime}$. Since $\left(h^{\ell}, W^{\ell}\right)$ satisfies the system of equations (4.14) for every integer $\ell$, we 
have for all $Z \in \mathbb{C}^{N}$ sufficiently close to the origin

$$
\sum_{r=0}^{k_{s}} P_{r, s}\left(Z, W^{\ell}(u)\right)\left(h_{s}^{\ell}(Z)\right)^{r}=0, s=1, \ldots, N .
$$

Since $\mathcal{K}^{1}$ satisfies property (ii) of Lemma 4.1 , we therefore obtain

$$
\mathcal{K}^{1}\left(Z, \zeta, W^{\ell}(u), \overline{W^{\ell}}(v) ; r_{1}^{\prime}\left(h^{\ell}(Z), \overline{h^{\ell}}(\zeta)\right)\right) \equiv 0
$$

for $(Z, \zeta) \in \mathbb{C}^{2 N}$ close to the origin. In what follows, we shall restrict (4.17) to the complexification $\mathcal{M}$. Since $W^{\ell}$ satisfies the second equation of the system (4.14), Lemma 4.2 implies that $B_{\nu}^{1}\left(Z, \bar{Z}, W^{\ell}(u), \overline{W^{\ell}(u)}\right)=0$, for $0 \leq \nu<\nu_{0}^{1}$, and for all $Z=(z, w, u) \in M$ sufficiently close to the origin. Equivalently, we have for $(Z, \zeta) \in \mathcal{M}$ near 0

$$
B_{\nu}^{1}\left(Z, \zeta, W^{\ell}(u), \overline{W^{\ell}}(v)\right)=0, \quad 0 \leq \nu<\nu_{0}^{1} .
$$

Hence (4.16) to (4.18) imply that

$$
\sum_{\nu=\nu_{0}^{1}}^{\delta_{1}} B_{\nu}^{1}\left(Z, \zeta, W^{\ell}(u), \overline{W^{\ell}}(v)\right)\left(r_{1}^{\prime}\left(h^{\ell}(Z), \overline{h^{\ell}}(\zeta)\right)\right)^{\nu}=0, \quad(Z, \zeta) \in \mathcal{M}, \text { near } 0 .
$$

Using (4.15), (4.19) yields that for $(Z, \zeta) \in \mathcal{M}$ sufficiently close to 0 ,

$$
-B_{\nu_{0}^{1}}^{1}\left(Z, \zeta, W^{\ell}(u), \overline{W^{\ell}}(v)\right)
$$

$$
=\sum_{\nu=\nu_{0}^{1}+1}^{\delta_{1}} B_{\nu}^{1}\left(Z, \zeta, W^{\ell}(Z), \overline{W^{\ell}}(\zeta)\right)\left(r_{1}^{\prime}\left(h^{\ell}(Z), \overline{h^{\ell}}(\zeta)\right)\right)^{\nu-\nu_{0}^{1}} .
$$

Since $h$ sends a neighborhood of $M$ to $M^{\prime}$ and since, for every integer $\ell$, the mapping $h^{\ell}$ agrees with $h$ up to order $\ell$ at 0 , it follows that $\left.r_{1}^{\prime}\left(h^{\ell}(Z), \overline{h^{\ell}}(\zeta)\right)\right|_{\mathcal{M}}$ vanishes at least to order $\ell$ at the origin. Hence from $(4.20)$, the same property holds for $\left.B_{\nu_{0}^{1}}^{1}\left(Z, \zeta, W^{\ell}(u), \overline{W^{\ell}}(v)\right)\right|_{\mathcal{M}}$. But also $W^{\ell}$ agrees with $\mathcal{H}$ up to order $\ell$ at the origin, so for every integer $\ell$, the germ at 0 of the holomorphic function $\left.B_{\nu_{0}^{1}}^{1}(Z, \zeta, \mathcal{H}(u), \overline{\mathcal{H}}(v))\right|_{\mathcal{M}}$ vanishes at least up to order $\ell$. As a consequence,

$$
B_{\nu_{0}^{1}}^{1}(Z, \zeta, \mathcal{H}(u), \overline{\mathcal{H}}(v)) \equiv 0, \quad \text { for }(Z, \zeta) \in \mathcal{M} \text { near } 0
$$

which contradicts the definition of $\nu_{0}^{1}$ by (4.11). This completes the proof of Lemma 4.3 and therefore the proof of Theorem 4.1 . 


\section{Holomorphic foliations in CR manifolds}

The main goal of this section is to study the holomorphic foliation (with singularities) arising from the existence of holomorphic vector fields tangent to a real-analytic (resp. real-algebraic) CR submanifold in complex space. The main result of this section is given by Proposition 5.2 and shows that the holomorphic foliation of a real-algebraic holomorphically degenerate CR submanifold of $\mathbb{C}^{N}$ is in fact algebraic. The results of this section will be used to derive Theorem 1.5 from Theorem 4.1 in Section 6.

\subsection{The real-analytic case}

Let $M \subset \mathbb{C}^{N}$ be a real-analytic generic submanifold of $\mathrm{CR}$ dimension $n$ and codimension $d$ so that $N=n+d \geq 2$. In what follows, for a point $p \in \mathbb{C}^{N}$, we denote by $\mathbb{O}_{p}$ the ring of germs of holomorphic functions at $p$ and by $\mathbb{M}_{p}$ its quotient field of meromorphic functions at $p$; these are the stalks of the sheaf of holomorphic (resp. meromorphic) functions, which we will accordingly denote by $\mathbb{O}$ and $\mathbb{M}$, respectively.

A holomorphic (resp. meromorphic) vector field on an open set $U \subset \mathbb{C}^{N}$ is a holomorphic (resp. meromorphic) section of $T^{(1,0)} \mathbb{C}^{N}$ over $U$, i.e., an expression of the form

$$
X=\sum_{j=1}^{N} a_{j}(Z) \frac{\partial}{\partial Z_{j}}, \quad a_{j} \in \mathbb{O}(U)(\mathbb{M}(U) \text { respectively }) .
$$

Again the stalks of these sheaves at $p \in \mathbb{C}^{N}$, i.e., expressions of the form

$$
X=\sum_{j=1}^{N} a_{j}(Z) \frac{\partial}{\partial Z_{j}}, \quad a_{j} \in \mathbb{O}_{p}\left(\mathbb{M}_{p} \text { respectively }\right)
$$

will be referred to as germs of holomorphic (respectively meromorphic) vector fields. We identify the sheaf of holomorphic (resp. meromorphic) vector fields with $\mathbb{O}^{N}\left(\mathbb{M}^{N}\right.$, respectively), and the germs of holomorphic (resp. meromorphic) vector fields at a point $p \in \mathbb{C}^{N}$ with $\left(\mathbb{O}_{p}\right)^{N}\left(\operatorname{resp} .\left(\mathbb{M}_{p}\right)^{N}\right)$.

For $p \in M$, let $\mathbb{T}_{p}$ (resp. $\mathbb{S}_{p}$ ) be the set of all germs at $p$ of holomorphic (resp. meromorphic) vector fields that are tangent to $M . \mathbb{T}_{p}$ is an $\mathbb{O}_{p^{-}}$ submodule of the free module $\left(\mathbb{O}_{p}\right)^{N} ; \mathbb{S}_{p}$ carries the structure of a finite dimensional vector space over $\mathbb{M}_{p}$. As in [5], for all $p \in M$, define

$$
\lambda_{M}(p):=\operatorname{dim}_{\mathbb{M}_{p}} \mathbb{S}_{p} \in\{0, \ldots, N-1\} .
$$


We recall from [5, Section 4] a known characterization of $\lambda_{M}(p)$ through local coordinates. For an arbitrary point $p \in M$ we may choose (see e.g., [3]) local holomorphic coordinates $Z=(z, \eta) \in \mathbb{C}^{n} \times \mathbb{C}^{d}$, vanishing at $p$, such that $M$ is given near the origin by a vector-valued equation of the form

$$
\eta=\Theta(z, \bar{z}, \bar{\eta})
$$

where $\Theta=\Theta(z, \chi, \sigma)$ is a $\mathbb{C}^{d}$-valued holomorphic map near 0 satisfying the reality condition

$$
\Theta(z, \chi, \bar{\Theta}(\chi, z, \eta)) \equiv \eta
$$

and

$$
\Theta(z, 0, \eta)=\Theta(0, \chi, \eta)=\eta
$$

such coordinates are commonly referred to as normal coordinates for $M$ at $p$. If $M$ is furthermore assumed to be real-algebraic, we may choose these local holomorphic coordinates as well as the mapping $\Theta$ to be algebraic.

Recall also that such a choice of normal coordinates can be made for points $q \in M$ nearby $p$ in such a way that the mapping $\Theta$ depends realanalytically on $q$ (and real-algebraically if $M$ is real-algebraic, see [3]). We expand the mapping $\bar{\Theta}$ into a Taylor series as follows:

$$
\bar{\Theta}(\chi, z, \eta):=\sum_{\beta \in \mathbb{N}^{n}} \bar{\Theta}_{\beta}(z, \eta) \chi^{\beta} .
$$

In what follows, we keep the above notation and choice of coordinates for a given fixed point $p \in M$. We also write the coordinates $Z=\left(Z_{1}, \ldots, Z_{N}\right)$. We need to recall the following slight generalization of a known criterion (see e.g., [5, Lemma 4.5]).

Lemma 5.1. In the above setting, the followings holds :

(i) a germ of a holomorphic vector field $X=\sum_{j=1}^{N} a_{j}(Z) \frac{\partial}{\partial Z_{j}}$ with $a_{j} \in \mathbb{O}_{0}$ is tangent to $M$ if and only if

$$
\sum_{j=1}^{N} a_{j}(Z) \frac{\partial \bar{\Theta}_{\beta}}{\partial Z_{j}}(Z) \equiv 0, \quad \text { for all } \beta \in \mathbb{N}^{n}
$$

the same holds for germs of meromorphic vector fields with $\mathbb{O}_{0}$ replaced by $\mathbb{M}_{0}$. 
(ii) there exists a neighborhood $U$ of 0 in $\mathbb{C}^{N}$ such that for every point $q \in M \cap U$, a germ of a holomorphic vector field $Y=\sum_{j=1}^{N} b_{j}(Z) \frac{\partial}{\partial Z_{j}}$ with $b_{j} \in \mathbb{O}_{q}$ is tangent to $M$ (near $\left.q\right)$ if and only if

$$
\sum_{j=1}^{N} b_{j}(Z) \frac{\partial \bar{\Theta}_{\beta}}{\partial Z_{j}}(Z) \equiv 0, \quad \text { for all } \beta \in \mathbb{N}^{n}
$$

for all $Z \in \mathbb{C}^{N}$ sufficiently close to $q$.

Proof. It is enough to prove (ii). We fix a polydisc $U_{1} \subset \mathbb{C}^{n}$ and $U_{2} \subset \mathbb{C}^{d}$ both containing the origin such that $\bar{\Theta}$ is holomorphic in $U_{1} \times U_{1} \times U_{2}$. Let $U:=U_{1} \times U_{2}$. Given $q=\left(z_{q}, \eta_{q}\right) \in M \cap U$, it is easy to see that a germ at $q$ of a holomorphic vector field $Y$ is tangent to $M$ near $q$ if and only $Y(\bar{\Theta}(\chi, Z))=0$ for all $Z$ in some connected neighborhood $U_{q} \subset \mathbb{C}^{N}$ of $q$ and for all $\chi$ in some neighborhood $\widetilde{U}_{1} \subset \mathbb{C}^{n}$ of $\bar{z}_{q}$. Since

$$
Y(\bar{\Theta}(\chi, Z))=\sum_{j=1}^{N} b_{j}(Z) \frac{\partial \bar{\Theta}}{\partial Z_{j}}(\chi, Z)
$$

the map $Y(\bar{\Theta}(\chi, Z))$ is in fact holomorphic in $U_{1} \times U_{q}$ and vanishes on $\widetilde{U}_{1} \times$ $U_{q}$. Hence $Y(\bar{\Theta}(\chi, Z))$ vanishes identically in $U_{1} \times U_{q}$. From this, the desired conclusion (5.7) follows.

We have the following result (see [5]).

Lemma 5.2. Let $M \subset \mathbb{C}^{N}$ be a real-analytic generic submanifold with $N \geq 2, p \in M$, and $(z, \eta)$ normal coordinates for $M$ near $p$. Then the following identity holds:

$$
\lambda_{M}(p)+r_{M}(p)=N
$$

where $r_{M}(p)$ is the generic rank of the holomorphic map $\left(\mathbb{C}^{N}, 0\right) \ni(z, \eta) \mapsto$ $\left(\bar{\Theta}_{\beta}(z, \eta)\right)_{\beta \in \mathbb{N}^{n}}$ defined by (5.5).

Proof. Choose an integer $\ell_{0}$ large enough so that the generic rank of the mapping $(z, \eta) \mapsto\left(\bar{\Theta}_{\beta}(z, \eta)\right)_{|\beta| \leq \ell_{0}}$ equals $r_{M}(p)$. Consider the $\mathbb{M}_{0}$ linear mapping 
$\mathcal{L}:\left(\mathbb{M}_{0}\right)^{N} \rightarrow\left(\mathbb{M}_{0}\right)^{c_{0}}$ given by

$$
\mathcal{L}\left(a_{1}, \ldots, a_{N}\right):=\left(\sum_{j=1}^{N} a_{j} \frac{\partial \bar{\Theta}_{\beta}}{\partial Z_{j}}\right)_{|\beta| \leq \ell_{0}}
$$

where $c_{0}=\operatorname{Card}\left\{\beta \in \mathbb{N}^{n}:|\beta| \leq \ell_{0}\right\}$. By Lemma 5.1 (i), $\mathbb{S}_{0}=\operatorname{Ker} \mathcal{L}$ and therefore, by the rank theorem, $N=\lambda_{M}(0)+\operatorname{dim}_{\mathbb{M}_{0}} \operatorname{Im} \mathcal{L}$. But the rank of the $\mathbb{M}_{0}$-linear mapping $\mathcal{L}$ coincides with the rank of the matrix $\left(\frac{\partial \bar{\Theta}_{\beta}}{\partial Z_{j}}\right)_{\substack{1 \leq j \leq N,|\beta| \leq \ell_{0}}}$ with entries in the field $\mathbb{M}_{0}$. This rank is exactly the generic rank of the (germ of the) holomorphic map $(z, \eta) \mapsto\left(\bar{\Theta}_{\beta}(z, \eta)\right)_{|\beta| \leq \ell_{0}}$. The proof of Lemma 5.2 is complete.

A first consequence of Lemma 5.2 is that $r_{M}(p)$ is independent of the choice of normal coordinates. Another useful consequence of Lemma 5.2 is the following (see [5] for the hypersurface case).

Lemma 5.3. Let $M \subset \mathbb{C}^{N}$ be a real-analytic generic submanifold. Then the functions $\lambda_{M}$ and $r_{M}$ are constant on any connected component of $M$.

Proof. Pick an arbitrary point $p \in M$. We first note that the definition of $\lambda_{M}$ implies that $\lambda_{M}$ is lower semi-continuous. Next, let $r_{M}(p)$ be as defined in Lemma 5.2. Since we may choose normal coordinates for points $q \in M$ nearby $p$ in such a way that the mapping $\Theta$ depends real-analytically on $q$, it follows that for all points $q$ sufficiently close to $p, r_{M}(q) \geq r_{M}(p)$. Hence by (5.8), we have that $\lambda_{M}(q) \leq \lambda_{M}(p)$ for all such $q$ 's. This latter fact together with the lower semi-continuity of $\lambda_{M}$ implies that $\lambda_{M}$ is constant in a neighborhood of $p$. Since the choice of $p$ is arbitrary, we obtain the desired statement.

In what follows, given a positive integer $r$, we shall denote by $\mathcal{E}_{h}^{r}$ the set of all germs through the origin in $\mathbb{C}^{r}$ of holomorphically nondegenerate realanalytic CR submanifolds. Recall also that given a positive integer $r$ and two germs of real submanifolds $(M, p)$ and $\left(M^{\prime}, p^{\prime}\right)$ in $\mathbb{C}^{r}$ we write $\left(M_{1}, p\right) \sim_{h}$ $\left(M_{2}, p^{\prime}\right)$ if there exists a germ of a biholomorphism of $\mathbb{C}^{r}$ at $p$ which maps the germ $(M, p)$ to $\left(M^{\prime}, p^{\prime}\right)$.

The following result provides a precise description of the holomorphic foliation on a (holomorphically degenerate) real-analytic CR submanifold. 
Proposition 5.1. Let $M \subset \mathbb{C}^{N}$ be a connected real-analytic $C R$ submanifold with $N \geq 2$. Then there exists a well-defined integer $\lambda_{M} \in\{0, \ldots$, $N-1\}$ and a closed proper real-analytic subvariety $\Upsilon_{M} \subset M$ such that

$$
M \backslash \Upsilon_{M}=\left\{p \in M:(M, p) \sim_{h}\left(\mathbb{C}^{\lambda_{M}} \times \widetilde{M}, 0\right), \text { where } \widetilde{M} \in \mathcal{E}_{h}^{N-\lambda_{M}}\right\}
$$

Furthermore, the real-analytic subvariety $\Upsilon_{M}$ is locally given by the intersection of germs of complex-analytic subvarieties with $M$.

Proof. We first treat the case where $M$ is a generic submanifold of $\mathbb{C}^{N}$. Let $\lambda_{M} \in\{0, \ldots, N-1\}$ be the integer defined by (5.1) and Lemma 5.3. Set

$$
\Omega_{M}^{h}:=\left\{p \in M:(M, p) \sim_{h}\left(\mathbb{C}^{\lambda_{M}} \times \widetilde{M}, 0\right) \text {, where } \widetilde{M} \in \mathcal{E}_{h}^{N-\lambda_{M}}\right\}
$$

In what follows we shall say that a point $p \in M$ satisfies property ( $)$ if there exists $Y_{1}, \ldots, Y_{\lambda_{M}} \in \mathbb{T}_{p}$ and a sufficiently small neighborhood $W$ of $p$ in $\mathbb{C}^{N}$ such that these $\lambda_{M}$ holomorphic vector fields are defined and linearly independent at every point $q \in W$. We first note that if a point $p \in \Omega_{M}$ then clearly $p$ satisfies property $(\boldsymbol{\Lambda})$. Conversely, if $p$ satisfies property $(\boldsymbol{A})$, then by straigthening the flows of $Y_{1}, \ldots, Y_{\lambda_{M}}$, we see that the germ $(M, p)$ is biholomorphically equivalent to the germ at the origin of a submanifold of the form $\mathbb{C}^{\lambda_{M}} \times \widetilde{M}$ where $\widetilde{M}$ is a germ through the origin of a real-analytic generic submanifold. Furthermore, $\widetilde{M}$ must necessarily be holomorphically nondegenerate since otherwise we could find $\lambda_{M}+1$ holomorphic vector fields tangent to $M$ near $p$ and generically linearly independent in a neighborhood of $p$, which contradicts the definition of $\lambda_{M}$.

Pick an arbitrary point $p \in M$. Since the ring $\mathbb{O}_{p}$ is noetherian, it follows that $\mathbb{T}_{p}$ is a finitely generated submodule of $\left(\mathbb{O}_{p}\right)^{N}$. Hence, there exists a connected neighborhood $U$ of $p$ in $\mathbb{C}^{N}$ and $r$ holomorphic vector fields $X_{1}, \ldots, X_{r}$ defined in $U$ such that $\mathbb{T}_{p}$ is generated by the germs at $p$ of the vector fields $X_{1}, \ldots, X_{r}$. In fact, we need a stronger property than that, and using Lemma 5.1 (ii) and Oka's theorem (see e.g., [12, Theorem 6.4.1]), it is possible (after shrinking $U$ if necessary) to assume that for every point $q \in$ $M \cap U$, the germs at $q$ of the vector fields $X_{1}, \ldots, X_{r}$ generate $\mathbb{T}_{q}$. We also may assume that $U$ is chosen sufficiently small so that $M \cap U$ is connected. It is not difficult to see that the generic rank of $\left(X_{1}, \ldots, X_{r}\right)$ over $U$ equals $\lambda_{M}$ as defined above. Setting $\mu(q)=\operatorname{Rk}\left(X_{1}(q), \ldots, X_{r}(q)\right)$ for all $q \in M \cap U$, we now claim that

$$
\{q \in M \cap U: q \text { satisfies }(\mathbf{A})\}=\left\{q \in M \cap U: \mu(q)=\lambda_{M}\right\}
$$


Note that we trivially have $\left\{q \in M \cap U: \mu(q)=\lambda_{M}\right\} \subset\{q \in M \cap U$ : $q$ satisfies ( $)\}$. Conversely, if $q \in M \cap U$ satisfies (\$), there exists $Y_{1}, \ldots, Y_{\lambda_{M}} \in \mathbb{T}_{q}$ and a sufficiently small neighborhood $W \subset U$ of $q$ in $\mathbb{C}^{N}$ such that these $\lambda_{M}$ holomorphic vector fields are defined and linearly independent at every point $q \in W$. Since the germs at $q$ of $X_{1}, \ldots, X_{r}$ generate the $\mathbb{O}_{q}$-module $\mathbb{T}_{q}$ and since $Y_{1}, \ldots, Y_{\lambda_{M}}$ are linearly independent at every point $q \in W$, it follows that the rank of $\left(X_{1}, \ldots, X_{r}\right)$ equals $\lambda_{M}$ at every point of $W$ and hence at $q$. This shows the claim (5.9).

Using the first part of the proof, we therefore obtain that $\Omega_{M}^{h} \cap U=$ $\left\{q \in M \cap U: \mu(q)=\lambda_{M}\right\}$ and hence $\Upsilon_{M} \cap U$ is given by the vanishing of a finite number of real-analytic functions on $U$ (namely the restriction to $M \cap U$ of the minors of size $\lambda_{M}$ of the Jacobian matrix of $\left.\left(X_{1}, \ldots, X_{r}\right)\right)$. This shows that $\Upsilon_{M}$ is a closed proper real-analytic subvariety of $M$ locally defined by the intersection of $M$ with a complex-analytic subvariety.

The case where $M$ is not generic follows from the generic case, after noticing that there exists an integer $s \in\{1, \ldots, N-1\}$ such that for every point $p_{0} \in M$, the germ $\left(M, p_{0}\right)$ is locally biholomorphically equivalent to a germ at the origin of a submanifold of the form $\{0\} \times M_{1} \subset \mathbb{C}^{s} \times \mathbb{C}^{N-s}$ with $M_{1}$ being a real-analytic generic submanifold in $\mathbb{C}^{N-s}$. We leave the remaining details to the reader. The proof of Proposition 5.1 is complete.

Remark 5.1. It is clear that the integer $\lambda_{M}$ in Proposition 5.1 is unique and may be defined as follows. If $M$ is as in Proposition 5.1, for every point $p \in M$, let $\mathcal{X}_{p} \subset \mathbb{C}^{N}$ be the intrinsic complexification of $M$ at $p$ (see e.g., [3]). Then $\mathcal{X}_{p}$ is the germ at $p$ of a complex submanifold (of smallest dimension) containing the germ of $M$ at $p$. Consider the field $\mathcal{K}_{p}$ of germs at $p$ of meromorphic functions in $\mathcal{X}_{p}$ and $\mathcal{S}_{p}$ the $\mathcal{K}_{p}$ vector space of all germs at $p$ of meromorphic vector fields of $\mathcal{X}_{p}$ tangent to $M$. Then it follows from Lemma 5.3 that $M \ni p \mapsto \operatorname{dim}_{\mathcal{K}_{p}} \mathcal{S}_{p}$ is constant and is the desired integer $\lambda_{M}$.

\subsection{The real-algebraic case}

We shall now establish the algebraic version of Proposition 5.1 when the submanifold $M$ is furthermore assumed to be real-algebraic. Analogously to the real analytic case, for a given positive integer $r$, we denote by $\mathcal{E}_{a}^{r}$ the set of all germs through the origin in $\mathbb{C}^{r}$ of holomorphically nondegenerate real-algebraic CR submanifolds. We also recall that given two germs of real submanifolds $(M, p)$ and $\left(M^{\prime}, p^{\prime}\right)$ in $\mathbb{C}^{r}$, we write $(M, p) \sim_{a}\left(M^{\prime}, p^{\prime}\right)$ if there exists a germ of an algebraic biholomorphism of $\mathbb{C}^{r}$ at $p$ which sends the germ $(M, p)$ to $\left(M^{\prime}, p^{\prime}\right)$. 
We are now ready to state the algebraic version of Proposition 5.1.

Proposition 5.2. Let $M \subset \mathbb{C}^{N}$ be a connected real-algebraic CR submanifold, $N \geq 2$. Let $\lambda_{M}$ and $\Upsilon_{M}$ be the associated integer and real-analytic subvariety of $M$ given by Proposition 5.1. Then $\Upsilon_{M}$ is in fact a proper realalgebraic subvariety of $M$ and the following holds:

$$
M \backslash \Upsilon_{M}=\left\{p \in M:(M, p) \sim_{a}\left(\mathbb{C}^{\lambda_{M}} \times \widetilde{M}, 0\right), \text { where } \widetilde{M} \in \mathcal{E}_{a}^{N-\lambda_{M}}\right\}
$$

Furthermore, the real-algebraic subvariety $\Upsilon_{M}$ is locally given by the intersection of germs of complex-algebraic subvarieties with $M$.

In order to prove Proposition 5.2, we shall need the following observation.

Lemma 5.4. Let $M$ be a germ of a real-algebraic $C R$ submanifold in $\mathbb{C}^{N}$ and $M^{\prime}$ be a germ of a real-analytic $C R$ submanifold in $\mathbb{C}^{N^{\prime}}$, both through the origin, with $1 \leq N^{\prime}<N$. Assume that

$$
(M, 0) \sim_{h}\left(M^{\prime} \times \mathbb{C}^{N-N^{\prime}}, 0\right)
$$

Then there exists a germ of a real-algebraic $C R$ submanifold $\widehat{M} \subset \mathbb{C}^{N^{\prime}}$ through the origin such that

$$
(M, 0) \sim_{a}\left(\widehat{M} \times \mathbb{C}^{N-N^{\prime}}, 0\right)
$$

Proof of Lemma 5.4. First note that if $M$ is not generic in $\mathbb{C}^{N}$, then there exists a positive integer $r \in\{1, \ldots, N-1\}$ and a real-algebraic generic submanifold $M_{1} \subset \mathbb{C}^{N-r}$ through the origin such that $(M, 0) \sim_{a}\left(\{0\} \times M_{1}, 0\right)$ (see e.g., [3]). From this fact, we see that we may assume in what follows that $M$ is generic in $\mathbb{C}^{N}$.

Let $n$ be the CR dimension of $M$ and $d$ its codimension. Choose normal coordinates $Z=\left(Z_{1}, \ldots, Z_{N}\right)=(z, \eta) \in \mathbb{C}^{n} \times \mathbb{C}^{d}$ as in Section 5.1, where $\Theta:\left(\mathbb{C}^{n+N}, 0\right) \rightarrow\left(\mathbb{C}^{d}, 0\right)$ is an algebraic holomorphic map of its arguments.

We first note that, by a usual straightening argument, (5.11) is equivalent to say that there exists a neighborhood $V$ of 0 in $\mathbb{C}^{N}$ and $N-N^{\prime}$ holomorphic vector fields $L_{1}, \ldots, L_{N-N^{\prime}}$ tangent to $M \cap V$ such that these $N-N^{\prime}$ vector fields are linearly independent at every point of $V$. Let $\varphi^{1}(t, Z)$ be the complex flow of the vector field $L_{1}$, flow that is defined for $(t, Z) \in \mathbb{C} \times \mathbb{C}^{N}$ sufficiently close to the origin. Recall that for sufficiently small $t,\left(\mathbb{C}^{N}, 0\right) \ni Z \mapsto \varphi^{1}(t, Z) \in\left(\mathbb{C}^{N}, 0\right)$ is a germ at 0 of a biholomorphism sending $(M, 0)$ to itself. Furthermore, writing $L_{1}=\sum_{j=1}^{N} a_{j}(Z) \frac{\partial}{\partial Z_{j}}$, 
we know that $\varphi^{1}=\left(\varphi_{1}^{1}, \ldots, \varphi_{N}^{1}\right)$ satisfies

$$
\frac{\partial \varphi_{j}^{1}}{\partial t}(t, Z)=a_{j}\left(\varphi^{1}(t, Z)\right), \quad j=1, \ldots, N, \quad \text { and } \varphi^{1}(0, Z)=Z
$$

for $(t, Z) \in \mathbb{C} \times \mathbb{C}^{N}$ sufficiently close to the origin. Since $L_{1}$ is tangent to $M$ (near 0), using the notation defined in (5.5) and Lemma 5.1 (i), we have

$$
\sum_{j=1}^{N} a_{j}\left(\varphi^{1}(t, Z)\right) \frac{\partial \bar{\Theta}_{\beta}}{\partial Z_{j}}\left(\varphi^{1}(t, Z)\right)=0, \quad \forall \beta \in \mathbb{N}^{n}
$$

for $(t, Z) \in \mathbb{C} \times \mathbb{C}^{N}$ sufficiently close to the origin. Combining (5.13) and (5.14), we obtain that near the origin in $\mathbb{C}^{N+1}$

$$
\frac{\partial}{\partial t}\left(\bar{\Theta}_{\beta}\left(\varphi^{1}(t, Z)\right)\right) \equiv 0, \quad \forall \beta \in \mathbb{N}^{n}
$$

We therefore have the following identity (that is contained, in the hypersurface case, in the statement of [5, Proposition 5.2])

$$
\bar{\Theta}_{\beta}\left(\varphi^{1}(t, Z)\right)=\bar{\Theta}_{\beta}(Z), \quad \forall \beta \in \mathbb{N}^{n},
$$

for all $(t, Z) \in \mathbb{C} \times \mathbb{C}^{N}$ sufficiently close to the origin. In what follows, we may assume without loss of generality that $a_{1}(0) \neq 0$. Consider now the $\mathbb{C}^{N}$-valued holomorphic mapping $\Psi$ defined in a neighborhood of the origin in $\mathbb{C}^{N}$ by $\Psi\left(w_{1}, \ldots, w_{N}\right)=\varphi^{1}\left(w_{1}, 0, w_{2}, \ldots, w_{N}\right)$. It is a standard fact that $\Psi$ is a local biholomorphism at the origin and that $\Psi_{*}\left(\frac{\partial}{\partial w_{1}}\right)=L_{1}$. Hence, since $L_{1}$ is tangent to $M$ near 0 , the vector field $\frac{\partial}{\partial w_{1}}$ is tangent to the germ of the real-analytic generic submanifold $\Psi^{-1}(M)$. Therefore the germ of $\Psi^{-1}(M)$ at 0 is of the form $\widetilde{M} \times \mathbb{C}$ where $\widetilde{M}$ is a germ at 0 of a real-analytic generic submanifold in $\mathbb{C}^{N-1}$. We now claim that $\widetilde{M}$ is in fact real-algebraic and that $(\widetilde{M} \times \mathbb{C}, 0) \sim_{a}(M, 0)$. To prove the claim, we note that it follows from (5.15) that for all $\beta \in \mathbb{N}^{n}, \bar{\Theta}_{\beta}(\Psi(w))=\bar{\Theta}_{\beta}\left(0, w_{2}, \ldots, w_{N}\right)=: C_{\beta}(w) \in$ $(\mathcal{N}\{w\})^{d}$. The system of algebraic equations

$$
\bar{\Theta}_{\beta}(Z)=C_{\beta}(w)
$$

has a convergent solution $Z=\Psi(w)$ and therefore, from an approximation theorem due to Artin [1], there exists an algebraic solution $\widehat{\Psi}:\left(\mathbb{C}^{N}, 0\right) \rightarrow$ $\left(\mathbb{C}^{N}, 0\right)$ of $(5.16)$ that agrees with $\Psi$ up to order one at 0 . Hence $\widehat{\Psi}$ is a local 
algebraic biholomorphism. Furthermore, since $\Psi$ sends $(\widetilde{M} \times \mathbb{C}, 0)$ to $(M, 0)$ and since for all $\beta \in \mathbb{N}^{n}$

$$
\bar{\Theta}_{\beta}(\Psi(w))=\bar{\Theta}_{\beta}(\widehat{\Psi}(w))
$$

it follows from $[4$, Lemma 14.3] that $\widehat{\Psi}$ sends also $(\widetilde{M} \times \mathbb{C}, 0)$ to $(M, 0)$, which proves the claim.

Since $(\widetilde{M} \times \mathbb{C}, 0) \sim_{a}(M, 0)$, there exists a neighborhood $W$ of 0 in $\mathbb{C}^{N-1}$ and $N-N^{\prime}-1$ holomorphic vector fields tangent to $\widetilde{M} \cap W$ such that these $N-N^{\prime}-1$ vector fields are linearly independent at every point of $W$. We can therefore apply the above reasoning to the real-algebraic generic submanifold $\widetilde{M} \subset \mathbb{C}^{N-1}$ and, by induction, we reach the desired conclusion.

Proof of Proposition 5.2. We note that if $\lambda_{M}=0$ (i.e., $M$ is holomorphically nondegenerate), there is nothing to prove, and therefore assume that $\lambda_{M}>$ 0 . Lemma 5.4 leads immediately to (5.10). It remains to show that $\Upsilon_{M}$ is a real-algebraic subvariety of $M$. In order to show this, we pick an arbitrary point $p \in M$ choose associated normal coordinates, in which $p=0$. We note that as in the proof of Proposition 5.1, we can choose a positive integer $\ell_{0}$ such that in a neighborhood $U$ of 0 , a germ at $q \in U$ of a holomorphic vector field is tangent to $M$ if and only if $\sum_{j=1}^{N} \frac{\partial \Theta_{\beta}}{\partial Z_{j}}(Z) a_{j}(Z)=0$ near $q$ for all $\beta$ with $|\beta| \leq \ell_{0}$. Thus, we have realized the holomorphic vector fields tangent to $M \cap U$ as a subsheaf of $\left.\mathbb{O}^{N}\right|_{U}$ given by the relations between the $\left(\frac{\partial \bar{\Theta}_{\beta}}{\partial Z_{1}}, \ldots, \frac{\partial \bar{\Theta}_{\beta}}{\partial Z_{N}}\right)$ with $|\beta| \leq \ell_{0}$. Oka's Theorem then implies that we can find a finite number of holomorphic generators of $\mathbb{T}_{0}$ which also generate $\mathbb{T}_{q}$ for $q$ near 0 .

We now claim that we can actually choose these generators algebraic if all the $\bar{\Theta}_{\beta}$ are algebraic; the remainder of the proof is then verbatim to the proof of Proposition 5.1.

First note that the sheaf of algebraic functions on $\mathbb{C}^{N}$ is coherent. This can be seen by applying the Weierstrass division theorem for algebraic power series in the proof of Oka's Theorem as found in e.g., [12]. In particular, the sheaf of algebraic relations between the $\left(\frac{\partial \bar{\Theta}_{\beta}}{\partial Z_{1}}, \ldots, \frac{\partial \bar{\Theta}_{\beta}}{\partial Z_{N}}\right)$ for $|\beta| \leq \ell_{0}$ is locally finitely generated, i.e., shrinking $U$ if necessary, there exist algebraic vector fields $\left(X_{1}, \ldots, X_{r}\right)$ defined over $U$ and tangent to $M$, such that every germ at a point $q \in M \cap U$ of an algebraic vector field tangent to $M$ near $q$ can be written as an algebraic linear combination of $X_{1}, \ldots, X_{r}$.

Now for every point $q \in U$, the ring of convergent power series $\mathbb{C}\{x-q\}$ centered at $q$ is a flat algebra over the ring of germs at $q$ of algebraic functions $\mathcal{N}\{x-q\}$. We can thus apply the "equational criterion for flatness" 
(see e.g.[15, Theorem 7.6]) to see that every $X \in \mathbb{T}_{q}$ can (in the sense of germs at $q$ ) be written as a linear combination with coefficients in $\mathbb{C}\{x-q\}$ of holomorphic vector fields tangent to $M$ with algebraic coefficients in $\mathcal{N}\{x-q\}$. Since we have already observed that all germs at $q$ of algebraic vector fields tangent to $M$ are generated by $X_{1}, \ldots, X_{r}$, we see that the claim is proved and, as noted before, this finishes the proof of Proposition 5.2.

Remark 5.2. The authors thank Clemens Bruschek for pointing out the simple proof by applying the flatness criterion in the second half of the proof of Proposition 5.2.

Remark 5.3. (i) In [7], the authors have considered another proper realalgebraic subvariety $\widetilde{\Upsilon}_{M}$ attached to any connected real-algebraic $\mathrm{CR}$ submanifold $M \subset \mathbb{C}^{N}$. This subvariety $\widetilde{\Upsilon}_{M}$ is defined as follows: $M \backslash \widetilde{\Upsilon}_{M}:=$ $\left\{p \in M:(M, p) \sim_{a}\left(\mathbb{C}^{\lambda_{M}} \times \widetilde{M}, 0\right)\right.$, where $\left.\widetilde{M} \in \widetilde{\mathcal{E}}_{a}^{N-\lambda_{M}}\right\}$, where $\widetilde{\mathcal{E}}_{a}^{N-\lambda_{M}}$ denotes the set of germs of all real-algebraic finitely nondegenerate realanalytic CR submanifolds in $\mathbb{C}^{N-\lambda_{M}}$ through the origin (see e.g., [3, 6] for the definition). Since we always have the strict inclusion $\widetilde{\mathcal{E}}_{a}^{N-\lambda_{M}} \subset \mathcal{E}_{a}^{N-\lambda_{M}}$, the subvariety $\Upsilon_{M}$ given by Proposition 5.2 is in general strictly smaller than $\widetilde{\Upsilon}_{M}$.

(ii) The reader should observe that the real-algebraic subvariety $\Upsilon_{M}$ given by Proposition 5.2 can also be defined as follows: $M \backslash \Upsilon_{M}$ consists of all points $p$ in $M$ for which there exists an integer $k, 0 \leq k \leq N-1$, such that $(M, p) \sim_{a}\left(\mathbb{C}^{k} \times \widetilde{M}, 0\right)$, where $\widetilde{M} \in \mathcal{E}_{h}^{N-k}$. Indeed, note that if there exists $k \in\{0, \ldots, N-1\}$ such that $(M, p) \sim_{h}\left(\mathbb{C}^{k} \times \widetilde{M}, 0\right)$ where $\widetilde{M} \in \mathcal{E}_{h}^{N-k}$, then we must necessarily have $k=\lambda_{M}$ in view of the definition of $\lambda_{M}$.

\section{Proof of Theorem 1.5}

By definition $\Sigma_{M}=\Sigma_{M}^{1} \cup \Sigma_{M}^{2}$ where $\Sigma_{M}^{1}$ is the set of points that are not of constant orbit dimension and $\Sigma_{M}^{2}$ is the set of points that are not regular for the foliation on $M$. By Proposition 5.2, $\Sigma_{M}^{2}$ is a closed proper real-algebraic subvariety of $M$ and since $\Sigma_{M}^{1}$ possesses clearly also the same property (see e.g. [3]), conclusion (i) of the theorem follows.

To prove (ii), we may assume that $M$ is a generic submanifold in $\mathbb{C}^{N}$ since the non-generic case can easily be reduced to the generic case. Let $p \in$ $M \backslash \Sigma_{M}$. Let also $M^{\prime} \subset \mathbb{C}^{N}$ be another real-algebraic generic submanifold, $p^{\prime} \in M^{\prime}$ and let $h:\left(\mathbb{C}^{N}, p\right) \rightarrow\left(\mathbb{C}^{N}, p^{\prime}\right)$ be a local biholomorphic map sending 
$M$ to $M^{\prime}$. If $M$ is holomorphically nondegenerate, the desired conclusion follows immediately from Theorem 4.1.

If not, there exists an integer $k \in\{1, \ldots, N-1\}$ such that $(M, p) \sim_{a}$ $\left(\widetilde{M} \times \mathbb{C}^{k}, 0\right)$ where $\widetilde{M}$ is a holomorphically nondegenerate real-algebraic generic submanifold through the origin in $\mathbb{C}^{N-k}$. Furthermore, since $\left(M^{\prime}, p^{\prime}\right) \sim_{h}(M, p)$, it follows from Lemma 5.4 that $\left(M^{\prime}, p^{\prime}\right) \sim_{a}\left(\widetilde{M}^{\prime} \times \mathbb{C}^{k}, 0\right)$, where $\widetilde{M}^{\prime}$ is a real-algebraic generic submanifold through the origin in $\mathbb{C}^{N}$ which is also necessarily holomorphically nondegenerate. In order to prove Theorem 1.5, we may therefore assume that $(M, p)=\left(\widetilde{M} \times \mathbb{C}^{k}, 0\right)$ and that $\left(M^{\prime}, p^{\prime}\right)=\left(\widetilde{M^{\prime}} \times \mathbb{C}^{k}, 0\right)$. We also write the mapping $h:\left(\mathbb{C}_{\widetilde{Z}}^{N-k} \times\right.$ $\left.\mathbb{C}_{\widehat{Z}}^{k}, 0\right) \rightarrow\left(\mathbb{C}_{\widetilde{Z}^{\prime}}^{N-k} \times \mathbb{C}_{\widehat{Z}^{\prime}}^{k}, 0\right)$, where $h(Z)=h(\widetilde{Z}, \widehat{Z})=\left(h_{1}(\widetilde{Z}, \widehat{Z}), h_{2}(\widetilde{Z}, \widehat{Z})\right) \in$ $\mathbb{C}^{N-k} \times \mathbb{C}^{k}$. We claim that $h_{1}$ is independent of $\widehat{Z}=\left(\widehat{Z}_{1}, \ldots, \widehat{Z}_{k}\right)$. Indeed, consider the holomorphic vector $V=h_{*}\left(\frac{\partial}{\partial \widehat{Z}_{j}}\right)$ where $j=1, \ldots, k$. We have

$$
V=\frac{\partial h_{1}}{\partial \widehat{Z}_{j}}\left(h^{-1}\left(Z^{\prime}\right)\right) \cdot \frac{\partial}{\partial \widetilde{Z}^{\prime}}+\frac{\partial h_{2}}{\partial \widehat{Z}_{j}}\left(h^{-1}\left(Z^{\prime}\right)\right) \cdot \frac{\partial}{\partial \widehat{Z}^{\prime}} .
$$

Since the vector field $\frac{\partial}{\partial \widehat{Z}_{j}}$ is tangent to $\widetilde{M} \times \mathbb{C}^{k}$ near $0, V$ is tangent to $\widetilde{M^{\prime}} \times \mathbb{C}^{k}$ near 0 . This implies that for every $\widehat{Z}^{\prime} \in \mathbb{C}^{k}$ sufficiently close to the origin, the holomorphic vector field in $\mathbb{C}^{N-k}$

$$
\frac{\partial h_{1}}{\partial \widehat{Z}_{j}}\left(h^{-1}\left(\widetilde{Z}^{\prime}, \widehat{Z}^{\prime}\right)\right) \cdot \frac{\partial}{\partial \widetilde{Z}^{\prime}}
$$

is tangent to $\widetilde{M^{\prime}}$ near 0 . Since $\widetilde{M^{\prime}}$ is holomorphically nondegenerate, we must necessarily have $\frac{\partial h_{1}}{\partial \widehat{Z}_{j}}\left(h^{-1}\left(\widetilde{Z}^{\prime}, \widehat{Z}^{\prime}\right)\right) \equiv 0$ near $0 \in \mathbb{C}^{N}$ for all $j=1, \ldots, k$, which proves the claim.

We can thus write $h(\widetilde{Z}, \widehat{Z})=\left(h_{1}(\widetilde{Z}), h_{2}(\widetilde{Z}, \widehat{Z})\right)$ where $h_{1}:\left(\mathbb{C}^{N-k}, 0\right) \rightarrow$ $\left(\mathbb{C}^{N-k}, 0\right)$ is a local biholomorphism sending $(\widetilde{M}, 0)$ to $\left(\widetilde{M^{\prime}}, 0\right)$. Since $\widetilde{M}$ is holomorphically nondegenerate and because the local $\mathrm{CR}$ orbits of $\widetilde{M}$ must also be of constant dimension in a neighborhood of 0 , we may apply Theorem 4.1 to conclude that for every integer $\ell$ there exists a local algebraic biholomorphism $h_{1}^{\ell}:\left(\mathbb{C}^{N-k}, 0\right) \rightarrow\left(\mathbb{C}^{N-k}, 0\right)$ sending $(\widetilde{M}, 0)$ to $\left(\widetilde{M}^{\prime}, 0\right)$ that agrees with $h_{1}$ up to order $\ell$ at 0 . For every integer $\ell$, define $h_{2}^{\ell}$ to be the $\ell$ th order Taylor polynomial of $h_{2}$ at 0 . Then the local algebraic biholomorphism $h^{\ell}:=\left(h_{1}^{\ell}, h_{2}^{\ell}\right)$ satisfies all the required conditions. The proof of Theorem 1.5 is complete. 


\section{Acknowledgments}

The authors would like to thank an anonymous referee for his helpful insights and comments, which helped us to essentially improve an earlier version of this paper.

The first author is supported by the Austrian Federal Ministry of Science and Research BMWF, START Prize Y377. The second author was partially supported by the French National Agency for Research (ANR), project DynPDE (programmes blancs). Both authors were partially supported by the Amadeus program of the Partenariat Hubert Curien and the FWF-ANR project CRARTIN

\section{References}

[1] M. Artin, Algebraic approximation of structures over complete local rings, Inst. Hautes Études Sci. Publ. Math. 36 (1969), 23-58.

[2] M.S. Baouendi, P. Ebenfelt and L.P. Rothschild, Algebraicity of holomorphic mappings between real algebraic sets in $\mathbb{C}^{n}$, Acta Math. 177 (1996), 225-273.

[3] M.S. Baouendi, P. Ebenfelt and L.P. Rothschild, Real submanifolds in complex space and their mappings, Princeton Math. Ser. 47, Princeton University Press, Princeton 1999.

[4] M.S. Baouendi, N. Mir and L.P. Rothschild, Reflection ideals and mappings between generic submanifolds in complex space, J. Geom. Anal. 12 (2002), 543-580.

[5] M.S. Baouendi and L.P. Rothschild, Mappings of real algebraic hypersurfaces, J. Amer. Math. Soc. 8 (1995), 997-1015.

[6] M.S. Baouendi, L.P. Rothschild and D. Zaitsev, Equivalences of real submanifolds in complex space, J. Differential Geom. 59 (2001), 301-351.

[7] M.S. Baouendi, L.P. Rothschild and D. Zaitsev, Points in general position in real-analytic submanifolds in $\mathbb{C}^{N}$ and applications, Complex analysis and geometry (Columbus, OH, 1999), Ohio State Univ. Math. Res. Inst. Publ., 9, de Gruyter, Berlin, 2001, 1-20.

[8] S. Berhanu, P. Cordaro and J. Hounie, An introduction to involutive structures, New Mathematical Monographs, 6, Cambridge University Press, Cambridge, 2008. 
[9] A. Boggess, CR manifolds and the tangential Cauchy-Riemann complex, Studies in Advanced Mathematics, CRC Press, Boca Raton, FL, 1991.

[10] A.M. Gabrielov, The formal relations between analytic functions, Funkcional. Anal. i Prilozen. 5 (1971), 64-65.

[11] X. Gong, Existence of real analytic surfaces with hyperbolic complex tangent that are formally but not holomorphically equivalent to quadrics, Indiana Univ. Math. J. 53 (2004), 83-95.

[12] L. Hörmander, An introduction to complex analysis in several variables, 3rd ed., North-Holland Mathematical Library, 7, North-Holland Publishing Co., Amsterdam, 1990.

[13] X. Huang and W. Yin, A Bishop surface with a vanishing Bishop invariant, Invent. Math. 176 (2009), 461-520.

[14] X. Huang and W. Yin, A codimension two CR singular submanifold that is formally equivalent to a symmetric quadric, Int. Math. Res. Not. 15 (2009), 2789-2828.

[15] H. Matsumura, Commutative Ring Theory, $2^{\text {nd }}$ ed. Cambridge Studies in Advanced Mathematics, 8, Cambridge University Press, Cambridge, 1989.

[16] F. Meylan, N. Mir and D. Zaitsev, Approximation and convergence of formal CR-mappings, Int. Math. Res. Not. 4 (2003), 211-242.

[17] N. Mir, Germs of holomorphic mappings between real-algebraic hypersurfaces, Ann. Inst. Fourier (Grenoble) 48(4) (1998), 1025-1043.

[18] N. Mir, On the convergence of formal mappings, Comm. Anal. Geom. 10 (2002), 23-59.

[19] H. Poincaré, Les fonctions analytiques de deux variables et la représentation conforme, Rend. Circ. Mat. Palermo 23 (1907), 185220.

[20] D. Popescu, General Néron desingularization and approximation, Nagoya Math. J. 104 (1986), 85-115.

[21] N. Stanton, Infinitesimal CR automorphisms of real hypersurfaces, Amer. J. Math. 118 (1996), 209-233.

[22] L. Stolovitch, Family of intersecting totally real manifolds of $\left(\mathbb{C}^{n}, 0\right)$ and CR-singularities. Preprint, 2005. 
[23] S.M. Webster, On the mapping problem for algebraic real hypersurfaces, Invent. Math. 43 (1977), 53-68.

FAKULtätT FÜR MATHEMATIK

UNIVERSITÄT WIEN

NORDBERGSTRASSE 15

A-1090 WIEN

ÖSTERREICH

E-mail address: bernhard.lamel@univie.ac.at

Laboratoire de Mathématiques Raphä̈L Salem

UNIVERSITÉ DE ROUEN

UMR 6085 CNRS

Avenue de L'Université, B.P. 12

76801 Saint Etienne DU RouvRay

FRANCE

E-mail address: nordine.mir@univ-rouen.fr

Received March 8, 2010 
